\title{
Natural and natural-like polyphenol compounds: in vitro antioxidant activity and potential for therapeutic application
}

\section{Type}

Research paper

\section{Keywords}

polyphenols, total antioxidant capacity, dehydrozingerone, hydroxylated biphenyls, trolox

\begin{abstract}
Introduction

Phenols are a large family of natural and synthetic compounds with known antioxidant activity. The aim of this study was to preform an in vitro screening of natural and natural-like phenol monomers and their C2-symetric dimers (hydroxylated biphenyls) in order to identify those representatives which pharmacophores have the strongest antioxidant and the lowest prooxidant activity.

\section{Material and methods}

Antioxidative properties of 36 compounds (monomers and their C2-symmetric dimers) were evaluated in vitro. Different (red/ox) assays were used to measure their total oxidative potential (TOP), their total antioxidative capacity (TAC), the pro-oxidative-antioxidant balance (PAB) and total $\mathrm{SH}$-group content $(\mathrm{SHG})$ in a biologically relevant environment. The Pro-oxidative Score, Antioxidative Score and the Oxy Score were also calculated. Trolox, a water soluble analogue of $\alpha-$ tocopherol was used as a positive control.
\end{abstract}

\section{Results}

In an assay consisting of pooled human serum 6 of the 36 compounds indicated significant antioxidant activity (compounds $6,7,12,13,26$, and 27 ) whereas 4 indicated extremely weak antioxidant activity (compounds 2, 29, 30, and 31). Within the 36 compounds comprising of zingerone, dehydrozingerone, aurone, chalcone, magnolol derivatives, in both monomeric and dimeric forms, the 2 compounds that indicated the highest antioxidant activity were dehydrozingerone derivatives (compounds 6 and 12). Trolox's activity was found between the strong and weak antioxidant compounds analysed in our study.

\section{Conclusions}

In this study selected dehydrozingerones were identified as good candidates for in-depth testing of their biological behaviour and for possible precursors for the synthesis of novel polyphenolic molecules with potential therapeutic applications. 


\begin{abstract}
Introduction: Phenols are a large family of natural and synthetic compounds with known antioxidant activity. The aim of this study was to preform an in vitro screening of natural and natural-like phenol monomers and their C2-symetric dimers (hydroxylated biphenyls) in order to identify those representatives which pharmacophores have the strongest antioxidant and the lowest prooxidant activity.
\end{abstract}

Material and Methods: Antioxidative properties of 36 compounds (monomers and their C2symmetric dimers) were evaluated in vitro. Different (red/ox) assays were used to measure their total oxidative potential (TOP), their total antioxidative capacity (TAC), the pro-oxidativeantioxidant balance (PAB) and total SH-group content (SHG) in a biologically relevant environment. The Pro-oxidative Score, Antioxidative Score and the Oxy Score were also calculated. Trolox, a water soluble analogue of $\alpha$-tocopherol was used as a positive control.

Results: In an assay consisting of pooled human serum 6 of the 36 compounds indicated significant antioxidant activity (compounds 6, 7, 12, 13, 26, and 27) whereas 4 indicated extremely weak antioxidant activity (compounds 2, 29, 30, and 31). Within the 36 compounds comprising of zingerone, dehydrozingerone, aurone, chalcone, magnolol derivatives, in both monomeric and dimeric forms, the 2 compounds that indicated the highest antioxidant activity were dehydrozingerone derivatives (compounds 6 and 12). Trolox's activity was found between the strong and weak antioxidant compounds analysed in our study.

Conclusion: In this study selected dehydrozingerones were identified as good candidates for in-depth testing of their biological behaviour and for possible precursors for the synthesis of novel polyphenolic molecules with potential therapeutic applications.

Key words: oxidative stress, polyphenols, dehydrozingerone, hydroxylated biphenyls, trolox 


\section{Introduction}

Phenols are a large family of natural and synthetic compounds with known antioxidant activity $[1,2]$. A deficit in antioxidant protection and/or excessive production of reactive oxygen species (ROS) in cells causes oxidative stress which is detrimental to living organisms [3]. Natural phenols are recognized as nutraceuticals, active components of functional food, often used as adjuvants in therapy, or in prevention of different diseases such as cardiovascular diseases [4], dyslipidemia [5], neurodegenerative diseases [6,7], and bacterial and viral infections. [8,9,10]

Despite the promising therapeutical and/or preventive effects and high safety profile, their use is limited, mainly because of their poor bioavailability. [4] Therefore, the use of sinthesized natural-like derivatives has potential to overcome this limitation.

So far, the most interest has been shown for derivatives of zingeron and curcumin. [11,12]. Zingerone [4-(4-hydroxy-3-methoxyphenyl)-2-butanone] is an active ingredient isolated from dried or heat-treated ginger (Zingiber officinale, family Zingiberaceae). Curcumin [1,7-bis(4hydroxy-3-methoxyphenyl)-1,6-heptadiene-3,5-dione] is an active component of the root of turmeric plant (Curcuma longa Linn, family Zingiberaceae).

Hydroxylated biphenyls are examples of natural plant-derived polyphenols. Due to their ability to bind to many types of proteins hydroxylated biphenyls could therefore affect biological processes within living organisms $[13,14]$. The most important pharmacophore in their structure consists of the two benzene rings bridged by a single covalent bond. The presence of steric hindrance caused by chemical groups positioned close to the single $\mathrm{C}-\mathrm{C}$ bond can lead to interactions between biphenyl structures and proteins via selective chiral recognition $[1,15$, 16]. A C2-symmetry axis in the structure allows the two aromatic rings indistinguishable. This axis facilitates the synthesis of compounds and their interactions with proteins. Due to their specific structure, hydroxylated biphenyls can reduce oxidative stress to an extent greater than both their corresponding natural and synthetic monophenols [17]. The antioxidative activity of hydroxylated biphenyls increases when the phenol hydroxyl groups are located in orthoposition to the single $\mathrm{C}-\mathrm{C}$ bond between the two aromatic rings as it influences intramolecular hydrogen bonding and stabilisation of the generated phenoxyl radical $[1,18]$. The presence of a methoxyl group in ortho position to the phenolic hydroxyl group (a guaiacyl unit) and an $\alpha, \beta$-unsaturated chain in 4 position provides even better stabilisation of the generated phenoxyl radical $[17,18]$.

Phenols, in addition to be antioxidants, can also exhibit pro-oxidant characteristics. Under normal conditions phenoxyl radicals formed during an antioxidative reaction are not pro- 
oxidative due to their rapid conversion back to non-radicals via polymerisation, enzymatic or non-enzymatic radical reduction reactions. However, phenoxyl radicals can exhibit cytotoxic pro-oxidative activity in the case of free radical life prolongation [19].

As phenols form the core in the structure of numerous drug molecules, diverse group of phenolic compounds, including natural and natural-like monomers and their $\mathrm{C} 2$-symetric dimers (hydroxylated biphenyls) was formed, and their effect on oxidative stress was investigated in this study. The antioxidative capability of selected monomers and their C2 symmetric dimers was determined using a number of in vitro assays. Moreover, the way different phenolic structures behave in biological matrix (blood serum) in regards to their interactions, conformational changes and formation of hydrogen bonds was analyzed. Many of the compounds were synthesized for the first time ever.

The goal of this study was to identify those representatives which pharmacophores have the strongest antioxidant and the lowest prooxidant activity in vitro conditions and thus conclude which phenolic pharmacophore is the most promising for potential further drug development, comparing its activities with Trolox, a hydrosoluble vitamin $\mathrm{E}$ analogue as proven antioxidant substance [20]

\section{Materials and Methods}

Reagents and solvents, were of analytical reagent grade and bought from Aldrich Chemie (Steinheimm, Germany) and Merck (Darmstadt, Germany).

Compounds 3 and 32 were purchased from Chemos GmbH (Regenstauf, Germany), compounds $\mathbf{2 2}$ and $\mathbf{2 4}$ were bought from Sigma Aldrich (Milan, Italy) and used without 
purification. Compounds 4 and 6-9 were prepared according procedures described by Marchiani et al. [21], compound $\mathbf{1 0}$ as described by Cook et al. [22], compound 12 as described by Choi et al. [23], compound 16 as described by Tatzuzaki et al. [24], compound 18 as described by Varro et al [25], compounds 20, 21 and 41 as described by Dettori et al [26], compound 42 as described by Oufensou et al [27], compound 33 as described by Lin et al. [28], compound $\mathbf{3 4}$ as described by Kong et al. [29], and compounds 35, 36 as described by Maioli et al. [15].

The purity of all new compounds was judged to be $>98 \%$ by ${ }^{1} \mathrm{H}$ NMR and ${ }^{13} \mathrm{C}$ NMR spectral determination.

Lipophilicity of compounds 1-36 was estimated by ChemBioDraw Ultra 13.0 software (Cambridge Soft) using the logarithm of the partition coefficient for $n$-octanol/water ( $\operatorname{LogP})$ and listed in Table I.

Analysis of antioxidant activity was performed by using a microplate reader (BioTek, Winooski, Vermont, USA) and ILAB 300+ automatic analyzer (Instrumentation Laboratory, Milan, Italy). Melting points were estimated with a Büchi 530 melting point apparatus in open capillaries and are uncorrected. All ${ }^{1} \mathrm{H}$ NMR and ${ }^{13} \mathrm{C}$ NMR spectra were acquired with a Varian VXR 5000 spectrometer at $399.94 \mathrm{MHz}$ and 75.42 MHz respectively; , all spectra were run at room temperature in $\mathrm{CDCl}_{3}$ solution (if not otherwise indicated). Chemical shifts are reported in $\mathrm{ppm}(\delta)$ on scale downfield from TMS as internal standard. Signal patterns are indicated as $\mathrm{s}$ (singlet), d (doublet), $\mathrm{t}$ (triplet), q (quartet), $\mathrm{m}$ (multiplet) or dd (double of doublets). Determination of elemental analyses was done using an elemental analyser Perkin-Elmer model 240 C. Acetone was distilled from $\mathrm{CaCl}_{2}$. Purification was achieved by silica gel column chromatography-using silica gel 60 (230-400 mesh, Kiesgel, EM Reagents) eluting with appropriate solution in the stated v:v proportions. Reaction progress was monitored by thin layer chromatography, $0.25 \mathrm{~mm}$ thick pre-coated silica plates (Polygram ${ }^{\circledR} S i l \mathrm{G} / \mathrm{UV}_{254}$, Macherey-Nagel) and spots were detected under UV light.

\section{Chemical synthesis of new compounds}

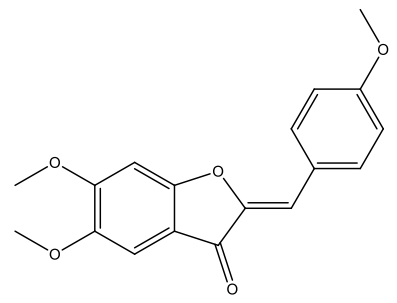


(Z)-5,6-dimethoxy-2-(4-methoxybenzylidene)benzofuran-3(2H)-one

Neutral alumina (13 g) is added to a solution of 4-methoxybenzaldehyde ( $0.88 \mathrm{~g}, 6.46 \mathrm{mmol})$ and 5,6-dimethoxybenzofuran-3(2H)-one [30] $(0.42 \mathrm{~g}, 2.15 \mathrm{mmol})$ in dichloromethane (10 $\mathrm{mL}$ ). The reaction mixture was thoroughly mixed at room temperature for $6 \mathrm{hrs}$. The solvent was rotoevaporated, to give the solid product which was purified by crystallization from dichloromethane-petroleum ether to afford 1 as a yellow solid $(0.41 \mathrm{~g}, 60 \%)$ : mp $199-200{ }^{\circ} \mathrm{C}$ (lit ${ }^{54}$ 199-201); ${ }^{1} \mathrm{H}$ NMR $\delta 3.86$ (s, 3H), 3.91 (s, 3H), 4.01 (s, 3H), 6.81 (s, Hz, Ar, 1H), 6.82 (s, $1 \mathrm{H}), 6.96(\mathrm{~d}, J=8.8 \mathrm{~Hz}, \mathrm{Ar}, 2 \mathrm{H}), 7.18(\mathrm{~s}, \mathrm{Ar}, 1 \mathrm{H}), 7.85(\mathrm{~d}, J=8.8 \mathrm{~Hz}, \mathrm{Ar}, 2 \mathrm{H}) ;{ }^{13} \mathrm{C}$ NMR $\delta$ 55.34, 56.31, 56.61, 95.51, 103.90, 112.29, 113.01, 114.37, 125.15, 133.15, 146.47, 146.88, 157.35, 160.78, 163.06, 183.10; Anal. Calcd. for $\mathrm{C}_{18} \mathrm{H}_{16} \mathrm{O}_{5}$ : C, 69.22; H, 5.16; Found: C, 69.33; H, 5.26 .

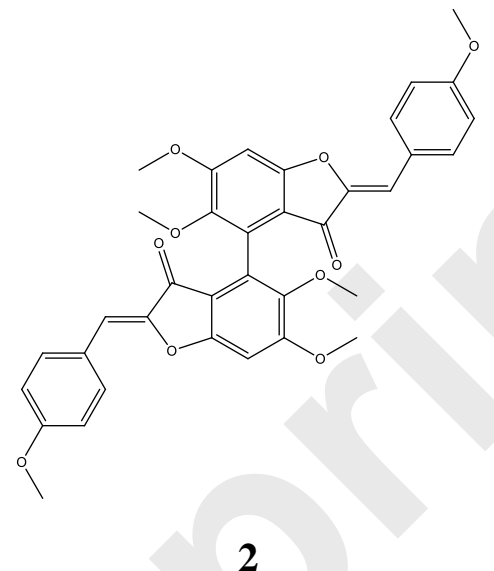

(2Z,2'Z)-5,5',6,6'-tetramethoxy-2,2'-bis(4-methoxybenzylidene)-[4,4'-bibenzofuran]$3,3^{\prime}\left(2 H, 2^{\prime} H\right)$-dione

Neutral alumina ( $2 \mathrm{~g})$ is added to a solution of 4-methoxybenzaldehyde $(0.13 \mathrm{~g}, 1 \mathrm{mmol})$ and compound $37(0.13 \mathrm{~g}, 0.33 \mathrm{mmol})$ in dichloromethane $(10 \mathrm{~mL})$. The reaction mixture was thoroughly mixed at room temperature for $6 \mathrm{hrs}$. Removal of the solvent yielded the solid product which was purified by crystallization from dichloromethane-petroleum ether to afford 2 as a yellow solid $(0.12 \mathrm{~g}, 59 \%)$ : mp $190-191{ }^{\circ} \mathrm{C} ;{ }^{1} \mathrm{H}$ NMR $\delta 3.63$ (s, 6H), $3.84(\mathrm{~s}, 6 \mathrm{H}), 4.01$ (s, 6H), $6.61(\mathrm{~s}, 2 \mathrm{H}), 6.91(\mathrm{~s}, \mathrm{Ar}, 2 \mathrm{H}), 6.94(\mathrm{~d}, J=8.4 \mathrm{~Hz}, \mathrm{Ar}, 4 \mathrm{H}), 7.80$ (d, J=8.4 Hz, Ar, 4H); ${ }^{13} \mathrm{C}$ NMR $\delta 55.33,56.35,61.06,96.07,111.61,112.41,114.32,124.97,125.35,132.98,143.51$, 146.91, 160.31, 160.59, 164.15, 182.23; Anal. Calcd. for $\mathrm{C}_{36} \mathrm{H}_{30} \mathrm{O}_{10}: \mathrm{C}, 69.45 ; \mathrm{H}, 4.86$; Found: C, 69.39; H, 4.96 .

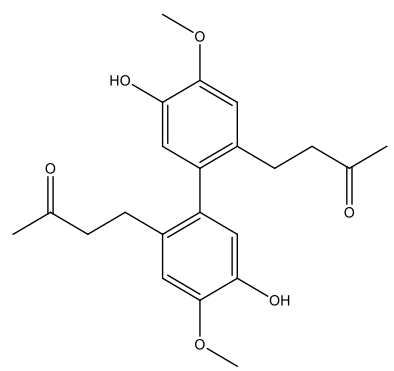


4,4'-(5,5'-dihydroxy-4,4'-dimethoxy-[1,1'-biphenyl]-2,2'-diyl)bis(butan-2-one)

To a solution of compound $38(0.21 \mathrm{~g}, 0.89 \mathrm{mmol})$ in dichloromethane $(15 \mathrm{~mL})$, a solution of molybdenum (V) chloride $(0.48 \mathrm{~g}, 1.78 \mathrm{mmol})$ in dichloromethane $(10 \mathrm{~mL})$ was added at $0{ }^{\circ} \mathrm{C}$ and under $\mathrm{N}_{2}$. The mixture was stirred at $0{ }^{\circ} \mathrm{C}$ for $45 \mathrm{~m}$. Water was cautiously added. The solution was extracted with dichloromethane and dried over $\mathrm{Na}_{2} \mathrm{SO}_{4}$. The crude product was purified by column chromatography using a 1:1 mixture of petroleum: acetone as eluent, to give 5 as a yellow oil (2.16 g, 60\%): ${ }^{1} \mathrm{H}$ NMR $\delta 1.99(\mathrm{~s}, 6 \mathrm{H}), 2.60-2.73$ (series of m, 8H), 3.87 (s, $6 \mathrm{H}), 6.62$ (s, Ar, 2H), 6.71 (s, Ar, 2H); ${ }^{13} \mathrm{C}$ NMR $\delta 27.05,29.87,45.12,55.91,111.34,116.18$, 130.62, 132.97, 143.38, 145.81, 208.35; Anal. Calcd.for $\mathrm{C}_{22} \mathrm{H}_{26} \mathrm{O}_{6}$ : C, 62.55; H, 5.14; Found: C, 62.54; H, 5.12.

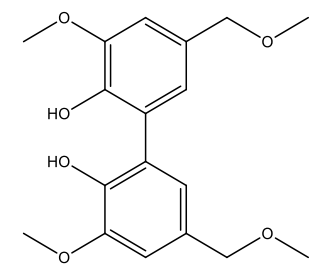

11

\section{3,3'-dimethoxy-5,5'-bis(methoxymethyl)-[1,1'-biphenyl]-2,2'-diol}

To a solution of $\mathbf{1 0}(1.6 \mathrm{~g}, 10.1 \mathrm{mmol})$ in dry dichloromethane (20 mL), methyltributylammonium permanganate (MTBAP) [31] (1.61 g, $5.05 \mathrm{mmol})$ in dry dichloromethane $(15 \mathrm{~mL})$ was added dropwise at room temperature and under $\mathrm{N}_{2}$. The solution was stirred at room temperature for $30 \mathrm{~min}$. An aqueous solution of $\mathrm{Na}_{2} \mathrm{~S}_{2} \mathrm{O}_{5}(50 \mathrm{~mL})$ was added. The organic layer was separated, washed with water, dried over $\mathrm{Na}_{2} \mathrm{SO}_{4}$ and evaporated to afford $\mathbf{1 1}$ as a white solid. Flash chromatography using a 1:2 mixture of ethyl acetate: petroleum ether as eluent gave 11 as white solid (1.03 g, 61\%): mp 103-104 ${ }^{\circ} \mathrm{C} ;{ }^{1} \mathrm{H}$ NMR $\delta 3.31$ (s, 6H), 3.87 (s, $6 \mathrm{H}), 5.37(\mathrm{~s}, 4 \mathrm{H}), 6.85(\mathrm{~d}, J=2 \mathrm{~Hz}, \mathrm{Ar}, 2 \mathrm{H}), 6.94(\mathrm{~d}, J=2 \mathrm{~Hz}, \mathrm{Ar}, 2 \mathrm{H}) ;{ }^{13} \mathrm{C} \mathrm{NMR} \delta 55.51$, 56.86, 74.20, 110.96, 122.93, 125.02, 129.39, 143.22, 147.73; Anal. Calcd. for $\mathrm{C}_{18} \mathrm{H}_{22} \mathrm{O}_{6}$ : C, 64.66; H, 6.63; Found: C, 64.76; H, 6.69.

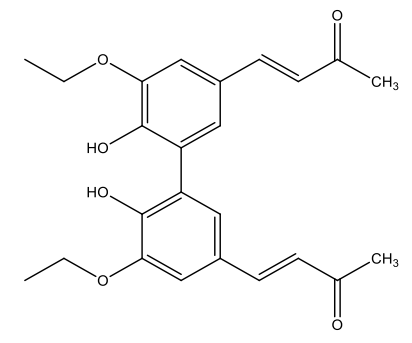

13

(3E,3'E)-4,4'-(5,5'-diethoxy-6,6'-dihydroxy-[1,1'-biphenyl]-3,3'-diyl)bis(but-3-en-2-one) 
An aqueous solution $(1 \mathrm{~N})$ of $\mathrm{LiOH}(40 \mathrm{~mL}, 40.0 \mathrm{mmol})$ was added dropwise to a stirred solution of $39(2.17 \mathrm{~g}, 6.6 \mathrm{mmol})$ in acetone $(50 \mathrm{~mL})$ at room temperature and under $\mathrm{N}_{2 . .}$ The mixture was stirred at $60^{\circ} \mathrm{C}$ for $12 \mathrm{hrs}, 10 \% \mathrm{HCl}$ and water were cautiously added. The resulting precipitate was filtered, washed with water and dried with $\mathrm{Na}_{2} \mathrm{SO}_{4}$. The product was purified by column chromatography using a 1:1 mixture of petroleum:ethyl acetate as eluent, to obtain 13 as a yellow solid $(2.16 \mathrm{~g}, 80 \%): \mathrm{mp}=100-101^{\circ} \mathrm{C} ;{ }^{1} \mathrm{H}$ NMR $\delta 1.50(\mathrm{t}, J=6.4 \mathrm{~Hz}, 6 \mathrm{H}), 2.35$ $(\mathrm{s}, 6 \mathrm{H}), 4.20(\mathrm{q}, J=6.4 \mathrm{~Hz}, 4 \mathrm{H}), 6.20(\mathrm{bs}, 2 \mathrm{H}), 6.59(\mathrm{~d}, J=16.4 \mathrm{~Hz}, 2 \mathrm{H}), 7.01(\mathrm{~d}, J=1.6 \mathrm{~Hz}$, Ar, 2H), 7.17 (d, $J=1.6 \mathrm{~Hz}, \mathrm{Ar}, 2 \mathrm{H}), 7.47$ (d, $J=16.4 \mathrm{~Hz}, 2 \mathrm{H}) ;{ }^{13} \mathrm{C}$ NMR $\delta 14.81,27.32,56.01$, 109.55, 123.56, 125.16, 125.28, 126.40, 143.69, 145.63, 146.55, 198.38; Anal. Calcd. for $\mathrm{C}_{24} \mathrm{H}_{26} \mathrm{O}_{6}:$ C, 70.23; H, 6.38; Found: C, 70.24; H, 6.32 .

\section{General procedure of the phenolic-OH group protection (compounds 14, 15, 17,19, 40)}

To a solution of phenol or biphenol (one equivalent) and $\mathrm{K}_{2} \mathrm{CO}_{3}$ (1.1 equivalents for monomer, 2.2 for dimers) in dry acetone, alkyl halide (methyl iodide or ethyl bromide or allyl bromide) (1.1 equivalents for monomer, 2.2 for dimers) was added under $\mathrm{N}_{2}$ at room temperature. The reaction mixture was stirred at $56^{\circ} \mathrm{C}$ for $12 \mathrm{hrs}$. Acetone was evaporated and $10 \% \mathrm{HCl}$ and water were added. The solution was extracted with diethyl ether, dried over $\mathrm{Na}_{2} \mathrm{SO}_{4}$ and evaporated. The crude was purified by flash chromatography to get the corresponding $O$ alkylated phenol.

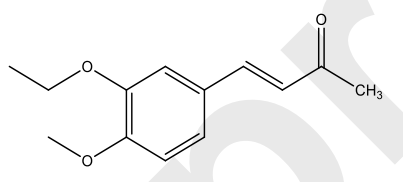

14

(E)-4-(3-ethoxy-4-methoxyphenyl)but-3-en-2-one

From 12, compound 14 was achieved after flash chromatography (ethyl acetate:petroleum ether 1:5) as a yellow solid $(0.42 \mathrm{~g}, 92 \%)$ : $\mathrm{mp} 145-147{ }^{\circ} \mathrm{C}$; ${ }^{1} \mathrm{H}$ NMR $\delta 1.49(\mathrm{t}, J=6.8 \mathrm{~Hz}, 3 \mathrm{H}), 2.35$ (s, 3H), $3.92(\mathrm{~s}, 3 \mathrm{H}), 4.13(\mathrm{q}, J=6.8 \mathrm{~Hz}, 2 \mathrm{H}), 6.61(\mathrm{~d}, J=16.4 \mathrm{~Hz}, 1 \mathrm{H}), 6.87(\mathrm{~d}, J=8.4 \mathrm{~Hz}$, Ar, 1H), 7.07 (d, $J=2 \mathrm{~Hz}, \mathrm{Ar}, 1 \mathrm{H}), 7.15(\mathrm{dd}, J=2,8.4 \mathrm{~Hz}, 1 \mathrm{H}), 7.45(\mathrm{~d}, J=16.4 \mathrm{~Hz}, 1 \mathrm{H}) ;{ }^{13} \mathrm{C}$ NMR $\delta$ 14.72, 27.33, 56.01, 64.36, 110.97, 111.23, 122.91, 125.17, 127.25, 143.66, 148.57, 151.62, 204.49; Anal. Calcd. for $\mathrm{C}_{13} \mathrm{H}_{16} \mathrm{O}_{3}: \mathrm{C}, 70.89$; H, 7.32; Found: C, 70.94; H, 7.36.

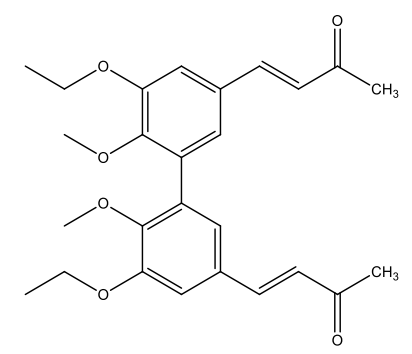

15

(3E,3'E)-4,4'-(5,5'-diethoxy-6,6'-dimethoxy-[1,1'-biphenyl]-3,3'-diyl)bis(but-3-en-2-one)

From 13, compound 15 was achieved after a flash chromatography (ethyl acetate:petroleum ether 1:1) as a yellow solid (0.84 g, 91\%): $\mathrm{mp} 141-142{ }^{\circ} \mathrm{C} ;{ }^{1} \mathrm{H}$ NMR $\delta 1.48(\mathrm{t}, J=7.2 \mathrm{~Hz}, 6 \mathrm{H})$, 
$2.34(\mathrm{~s}, 6 \mathrm{H}), 3.72(\mathrm{~s}, 6 \mathrm{H}), 4.13(\mathrm{q}, J=7.2 \mathrm{~Hz}, 4 \mathrm{H}), 6.62(\mathrm{~d}, J=16.4 \mathrm{~Hz}, 2 \mathrm{H}), 7.03(\mathrm{~d}, J=2 \mathrm{~Hz}$, Ar, 2H), $7.10(\mathrm{~d}, J=2 \mathrm{~Hz}, \mathrm{Ar}, 2 \mathrm{H}), 7.45(\mathrm{~d}, J=16.4 \mathrm{~Hz}, 2 \mathrm{H}) ;{ }^{13} \mathrm{C}$ NMR $\delta 14.88,27.47,60.81$, 64.47, 111.93, 124.05, 126.35, 129.66, 132.43, 143.21, 149.27, 152.31, 198.29; Anal. Calcd. for $\mathrm{C}_{26} \mathrm{H}_{30} \mathrm{O}_{6}$ : C, 71.21; H, 6.90; Found: C, 71.14; H, 6.96.

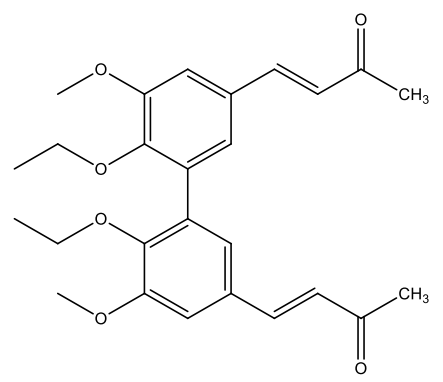

17

(3E,3'E)-4,4'-(6,6'-diethoxy-5,5'-dimethoxy-[1,1'-biphenyl]-3,3'-diyl)bis(but-3-en-2-one)

From 7, compound 17 was achieved after a flash chromatography (ethyl acetate:petroleum ether 1:2) as a yellow solid $(0.82 \mathrm{~g}, 89 \%)$ : $\mathrm{mp} 138-139{ }^{\circ} \mathrm{C} ;{ }^{1} \mathrm{H}$ NMR $\delta 1.09(\mathrm{t}, J=7.2 \mathrm{~Hz}, 6 \mathrm{H}), 2.36$ (s, 6H), 3.87-4.01 (series of m, 10H), $6.62(\mathrm{~d}, J=16 \mathrm{~Hz}, 2 \mathrm{H}), 7.10$ (m, Ar, 4H), 7.45 (d, $J=16$ $\mathrm{Hz}, 2 \mathrm{H}) ;{ }^{13} \mathrm{C}$ NMR $\delta 15.56,27.50,55.94,69.16,110.60,124.41,126.29,129.50,132.79$, 143.21, 148.39, 153.22, 198.29; Anal. Calcd. for $\mathrm{C}_{26} \mathrm{H}_{30} \mathrm{O}_{6}: \mathrm{C}, 71.21$; H, 6.90; Found: C, 71.29; H, 6.86 .

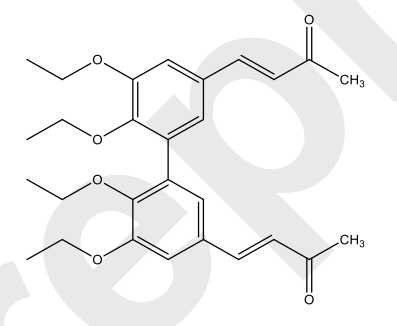

19

(3E,3'E)-4,4'-(5,5',6,6'-tetraethoxy-[1,1'-biphenyl]-3,3'-diyl)bis(but-3-en-2-one)

From 13, compound 19 was achieved after a flash chromatography (ethyl acetate:petroleum ether 2:3) as a yellow solid (0.78 g, 80\%): $\mathrm{mp} 143-144{ }^{\circ} \mathrm{C} ;{ }^{1} \mathrm{H}$ NMR $\delta 1.11(\mathrm{t}, J=7.2 \mathrm{~Hz}, 6 \mathrm{H})$, $1.49(\mathrm{t}, J=7.2 \mathrm{~Hz}, 6 \mathrm{H}), 2.35(\mathrm{~s}, 6 \mathrm{H}), 3.92(\mathrm{q}, J=7.2 \mathrm{~Hz}, 4 \mathrm{H}), 4.13(\mathrm{q}, J=7.2 \mathrm{~Hz}, 4 \mathrm{H}), 6.63$ $(\mathrm{d}, J=16 \mathrm{~Hz}, 2 \mathrm{H}), 7.08$ (d, $J=2.4 \mathrm{~Hz}, \mathrm{Ar}, 2 \mathrm{H}), 7.10$ (d, $J=2.4 \mathrm{~Hz}, \mathrm{Ar}, 2 \mathrm{H}), 7.44$ (d, $J=16$ $\mathrm{Hz}, 2 \mathrm{H}) ;{ }^{13} \mathrm{C}$ NMR $\delta 14.88,15.60,27.47,64.45,69.14,111.79,124.38,126.17,129.34,132.86$, 143.35, 148.65, 152.48, 198.32; Anal. Calcd. for $\mathrm{C}_{28} \mathrm{H}_{34} \mathrm{O}_{6}$ : C, 72.08; H, 7.35; Found: C, 72.14; H, 7.36.

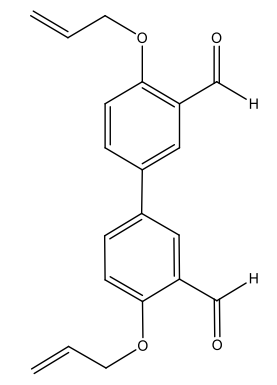




\section{4,4'-bis(allyloxy)-[1,1'-biphenyl]-3,3'-dicarbaldehyde}

From 25, compound $\mathbf{4 0}$ was achieved after a flash chromatography (acetone: petroleum ether 1:2) as a white solid $(0.64 \mathrm{~g}, 56 \%)$ : $\mathrm{mp} 162^{\circ} \mathrm{C} ;{ }^{1} \mathrm{H}$ NMR $\delta 4.82(\mathrm{~m}, 4 \mathrm{H}), 5.31$ (dd, $J=1.6 ; 12.4$ $\mathrm{Hz}, 2 \mathrm{H}), 5.51(\mathrm{dd}, J=1.6,18.8 \mathrm{~Hz}, 2 \mathrm{H}), 6.16(\mathrm{~m}, 2 \mathrm{H}), 7.31(\mathrm{~d}, J=9.2, \mathrm{Ar}, 2 \mathrm{H}), 7.92$ (dd, $J=$ 2.4, $9.2 \mathrm{~Hz}, \mathrm{Ar}, 2 \mathrm{H}), 7.98(\mathrm{~d}, J=2.4 \mathrm{~Hz}, \mathrm{Ar}, 2 \mathrm{H}), 10.54(\mathrm{~s}, 1 \mathrm{H}) ;{ }^{13} \mathrm{C}$ NMR $\delta$ 69.25, 114.30, 117.20, 125.23, 132.08, 133.00, 133.74, 160.36, 188.44, 205.27; Anal. Calcd. for $\mathrm{C}_{20} \mathrm{H}_{18} \mathrm{O}_{4}$ : C, 74.52; H, 5.63; Found: C, 74.56; H, 5.66.

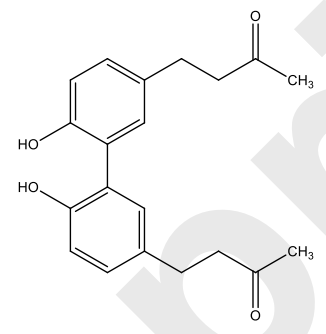

23

\section{4,4'-(6,6'-dihydroxy-[1,1'-biphenyl]-3,3'-diyl)bis(butan-2-one)}

Methyl-tributylammonium permanganate (MTBAP) [31] $(0.49 \mathrm{~g}, 1.5 \mathrm{mmol})$ in dry dichloromethane $(15 \mathrm{~mL})$ was added at room temperature, dropwise and under $\mathrm{N}_{2}$ to a solution of $22(0.5 \mathrm{~g}, 3 \mathrm{mmol})$ in dry dichloromethane $(20 \mathrm{~mL})$. Thereaction mixture was stirred at $20^{\circ} \mathrm{C}$ for $1 \mathrm{~h}$ and then was washed with an aqueous solution of $\mathrm{Na}_{2} \mathrm{~S}_{2} \mathrm{O}_{5}(50 \mathrm{~mL})$. The organic layer was separated, washed with water, dried over $\mathrm{Na}_{2} \mathrm{SO}_{4}$ and rotoevaporated to give as a brown solid. Purification by flash chromatography using a 1:1 mixture of ethyl acetate:petroleum ether as eluent gave 23 as white solid $(0.22 \mathrm{~g}, 46 \%)$ : mp 83-84 ${ }^{\circ} \mathrm{C} ;{ }^{1} \mathrm{H}$ NMR $\delta 2.14(\mathrm{~s}, 6 \mathrm{H}), 2.70$ 2.01 (series of m, 8H), $6.88(\mathrm{~d}, J=7.6 \mathrm{~Hz}, \mathrm{Ar}, 2 \mathrm{H}), 7.10(\mathrm{dd}, J=2.4,7.6 \mathrm{~Hz}, \mathrm{Ar}, 2 \mathrm{H}), 7.13$ (d, $J=2.4 \mathrm{~Hz}, \mathrm{Ar}, 2 \mathrm{H}) ;{ }^{13} \mathrm{C}$ NMR $\delta 28.08,30.15,45.35,116.96,125.24,129.21,131.22,133.63$, 151.21, 209.51; Anal. Calcd. for $\mathrm{C}_{20} \mathrm{H}_{22} \mathrm{O}_{4}$ : C, 73.60; H, 6.79; Found: C, 73.66; H, 6.74.<smiles>O=Cc1cc(-c2ccc(O)c(C=O)c2)ccc1O</smiles> 
4,4'-dihydroxy-[1,1'-biphenyl]-3,3'-dicarbaldehyde

A solution of 4,4'-biphenol $(0.20 \mathrm{~g}, 1.1 \mathrm{mmol})$ and hexamethylenetetramine $(0.2 \mathrm{~g}, 1.3 \mathrm{mmol})$ in TFA $(3 \mathrm{~mL})$ was heated at $100^{\circ} \mathrm{C}$ for $10 \mathrm{~min}$ under microwave irradiation $(100 \mathrm{~W})$. The reaction mixture was extracted with dichloromethane $(2 \times 30 \mathrm{~mL})$; the organic phase was washed with $\mathrm{NaHCO}_{3}$ solution, dried over $\mathrm{Na}_{2} \mathrm{SO}_{4}$ and evaporated. The crude product was washed with $\mathrm{EtOH}$ to eliminate impurities to give compound 25 as yellow solid $(0.16 \mathrm{~g}, 60 \%)$ : mp 106-107 ${ }^{\circ} \mathrm{C}\left(\right.$ Lit. $\left.^{53} 106-108{ }^{\circ} \mathrm{C}\right) ;{ }^{1} \mathrm{H}$ NMR (acetone d $\left.{ }_{6}\right) \delta 7.10(\mathrm{~d}, J=8.8 \mathrm{~Hz}, 2 \mathrm{H}), 7.93(\mathrm{dd}$, $J=2,4$ and $8.8 \mathrm{~Hz}, 2 \mathrm{H}), 8.11(\mathrm{~d}, J=2.4 \mathrm{~Hz}, 2 \mathrm{H}), 10.13(\mathrm{~s}, 2 \mathrm{H}) ;{ }^{13} \mathrm{C}$ NMR (acetone $\left.\mathrm{d}_{6}\right): \delta$ 117.83, 121.24, 131.22, 131.30, 134.80, 160.59, 197.09; Anal. Calcd. for $\mathrm{C}_{14} \mathrm{H}_{10} \mathrm{O}_{4}$ : C, 69.42; H, 4.16; Found: C, 69.45; H, 4.19.<smiles>CCC(C)(O)/C=C/c1ccccc1O</smiles>

26

(E)-2-(3-hydroxy-3-methylpent-1-en-1-yl) phenol

$\mathrm{Cs}_{2} \mathrm{CO}_{3}(0.47 \mathrm{~g}, 1.5 \mathrm{mmol}), \mathrm{Pd}(\mathrm{OAc})_{2}(0.06 \mathrm{~g}, 0.29 \mathrm{mmol})$ and 3-methylpent-1-en-3-ol (0.58 $\mathrm{g}, 5.8 \mathrm{mmol})$ were added to a solution of 2-bromophenol $(0.5 \mathrm{~g}, 2.9 \mathrm{mmol})$ in DMF $(10 \mathrm{~mL})$. The mixture was stirred under MW irradiation for $20 \mathrm{~min}$ at $100 \mathrm{~W}$ and $100^{\circ} \mathrm{C}$. The reaction mixture was diluted with $\mathrm{Et}_{2} \mathrm{O}$ and washed with brine. The organic layer was dried over $\mathrm{Na}_{2} \mathrm{SO}_{4}$, and rotoevaporated. The product was purified by flash-chromatography on silica gel using a 1:3 mixture of ethyl acetate: petroleum ether as eluent to give product $\mathbf{2 6}(0.23 \mathrm{~g}, 42 \%)$ : mp 108-109 ${ }^{\circ} \mathrm{C} ;{ }^{1} \mathrm{H}$ NMR (acetone $\left.\mathrm{d}_{6}\right) \delta 0.91(\mathrm{t}, J=7.6 \mathrm{~Hz}, 3 \mathrm{H}), 1.31(\mathrm{~s}, 3 \mathrm{H}) ; 1.62(\mathrm{q}, J=7.6$ $\mathrm{Hz}, 2 \mathrm{H}) ; 2.84(\mathrm{bs}, \mathrm{OH}) ; 6.31(\mathrm{~d}, J=16.4 \mathrm{~Hz}, 1 \mathrm{H}) ; 6.8(\mathrm{td}, J=0.8,7.2 \mathrm{~Hz}, 1 \mathrm{H}) ; 6.86(\mathrm{dd}, J=$ $0.8,8 \mathrm{~Hz}, 1 \mathrm{H}) ; 6.91(\mathrm{~d}, J=16.4 \mathrm{~Hz}, 1 \mathrm{H}) ; 7.03(\mathrm{td}, J=1.2$ and $8.0 \mathrm{~Hz}, 1 \mathrm{H}) ; 7.41(\mathrm{dd}, J=1.2,8$ $\mathrm{Hz}, 1 \mathrm{H}), 8.37$ (bs, OH); ${ }^{13} \mathrm{C}$ NMR $\delta 8.36,27.69,35.38,51.70,115.92,120.82,121.77,124.22$, 127.30, 28.51, 129.01, 138.04; Anal. Calcd. for $\mathrm{C}_{12} \mathrm{H}_{16} \mathrm{O}_{2}$ : C, 74.97; H, 8.39; Found: C, 74.88; $\mathrm{H}, 8.43$.

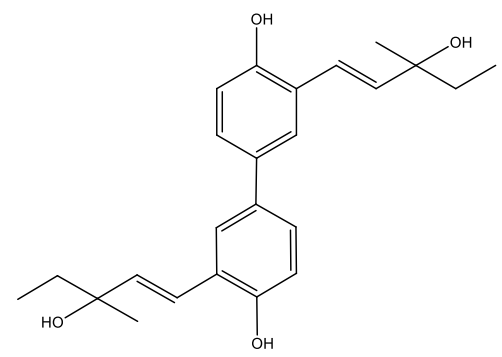

27

3,3'-bis((E)-3-hydroxy-3-methylpent-1-en-1-yl)-[1,1'-biphenyl]-4,4'-diol

A solution of compound $42(0.5 \mathrm{~g}, 1.45 \mathrm{mmol})$ in DMF (30 mL) $\mathrm{Cs}_{2} \mathrm{CO}_{3}(0.94 \mathrm{~g}, 2.9 \mathrm{mmol})$, $\mathrm{Pd}(\mathrm{OAc})_{2}(0.097 \mathrm{~g}, 0.145 \mathrm{mmol})$ and 3-methylpent-1-en-3-ol (0.58 g, $\left.5.8 \mathrm{mmol}\right)$ was stirred under MW irradiation at $100 \mathrm{~W}$ for $20 \mathrm{~min}$ and at $100^{\circ} \mathrm{C}$. The reaction mixture was then diluted 
with $\mathrm{Et}_{2} \mathrm{O}$ and washed with brine. The organic layer, dried over $\mathrm{Na}_{2} \mathrm{SO}_{4}$, was concentrated under vacuum. The product was purified by flash-chromatographed on silica gel eluting with 3:1 mixture of petroleum:ethylacetate to give product $27(0.2 \mathrm{~g}, 40 \%): \mathrm{mp} 130{ }^{\circ} \mathrm{C} ;{ }^{1} \mathrm{H}$ NMR(acetone $\left.\mathrm{d}_{6}\right) \delta 0.92(\mathrm{t}, J=7.2 \mathrm{~Hz}, 4 \mathrm{H}), 1.32(\mathrm{~s}, 6 \mathrm{H}), 1.62(\mathrm{q}, J=7.2 \mathrm{~Hz}, 6 \mathrm{H}), 3.52(\mathrm{bs}, \mathrm{OH}$, $2 \mathrm{H}), 6.47(\mathrm{~d}, J=16.0 \mathrm{~Hz}, 2 \mathrm{H}), 6.92(\mathrm{~d}, J=8.8 \mathrm{~Hz}, 2 \mathrm{H}), 7.28(\mathrm{dd}, J=2.0,8.8 \mathrm{~Hz}, 2 \mathrm{H}), 7.64(\mathrm{~d}$, $J=2.0 \mathrm{~Hz}$ ), 8.49 (bs, OH, 2H); ${ }^{13} \mathrm{C}$ NMR $\delta 7.89,27.37,35.42,72.35,115.94,121.61,124.65$, 124.73, 126.01, 132.79, 137.60, 153.55; Anal. Calcd. for $\mathrm{C}_{24} \mathrm{H}_{30} \mathrm{O}_{4}$ : C, 75.36; H, 7.91; Found: C, 75.38; H, 7.96.

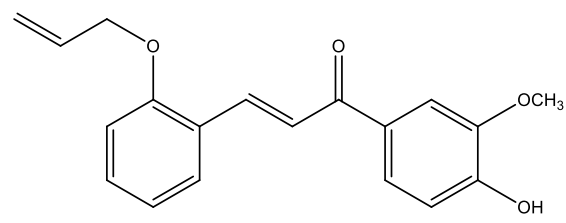

28

\section{(E)-3-(2-(allyloxy)phenyl)-1-(4-hydroxy-3-methoxyphenyl)prop-2-en-1-one}

A mixture of 2-(allyloxy)benzaldehyde [32] (0.20 g, 1,2 mmol), apocynin (0.2 g, $1.2 \mathrm{mmol})$ and $\mathrm{LiOH}(0.17 \mathrm{~g}, 7.4 \mathrm{mmol})$ in $\mathrm{MeOH}(5 \mathrm{ml})$ was subjected, in a $30 \mathrm{~mL}$ glass pressure microwave tube, equipped with a magnetic stirrer bar, to microwave irradiation (power: 100 $\mathrm{W}$; temperature: $70^{\circ} \mathrm{C}$ ) for $15 \mathrm{~min}$. The reaction mixture was then acidified with $\mathrm{HCl} 10 \%$ solution and extracted with dichloromethane $(2 \times 30 \mathrm{~mL})$. The organic solution was dried over $\mathrm{Na}_{2} \mathrm{SO}_{4}$ and roto evaporated. The product was purified by flash chromatography using a 4:1 petroleum ether:acetone mixture as eluent, to obtain compound $\mathbf{2 8}$ as a yellow solid. $(0.2 \mathrm{~g}$, 53\%): $\mathrm{mp} 94^{\circ} \mathrm{C} ;{ }^{1} \mathrm{H}$ NMR $\delta 3.96(\mathrm{~s}, 3 \mathrm{H}), 4.63(\mathrm{~d}, J=5.2 \mathrm{~Hz}, 2 \mathrm{H}), 5.44(\mathrm{dd}, J=1.2,17.2 \mathrm{~Hz}$, 2H), $6.10(\mathrm{~m}, 1 \mathrm{H}), 6.91(\mathrm{~d}, J=8.0 \mathrm{~Hz}, \mathrm{Ar}, 1 \mathrm{H}), 6.97(\mathrm{~m}, \mathrm{Ar}, 2 \mathrm{H}), 7.34(\mathrm{t}, J=1.6 \mathrm{~Hz}, \mathrm{Ar}, 1 \mathrm{H})$, $7.65(\mathrm{~m}, \mathrm{Ar}, 3 \mathrm{H}), 7.71(\mathrm{~d}, J=16 \mathrm{~Hz}, 1 \mathrm{H}), 8.14(\mathrm{~d}, J=16 \mathrm{~Hz}, 1 \mathrm{H}) .{ }^{13} \mathrm{C}$ NMR $\delta 56.10,69.10$, $110.53,112.49$, 113.72, 117.99, 120.94, 122.72, 123.64, 124.34, 129.57, 131.25, 131.41, 132.87, 139.67, 146.49, 150.19, 157.75, 189.22; Anal. Calcd. for $\mathrm{C}_{19} \mathrm{H}_{18} \mathrm{O}_{4}: \mathrm{C}, 73.53 ; \mathrm{H}, 5.85$; Found: C, 73.56; H, 5.86 .

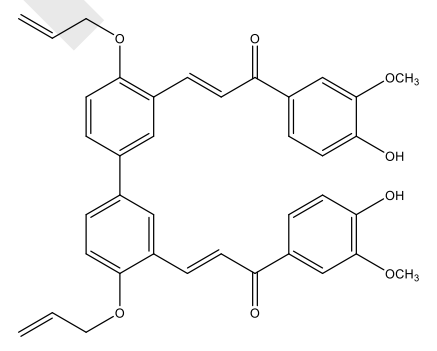

29

(E)-3-(4,4'-bis(allyloxy)-3'-((E)-3-(3-hydroxy-4-methoxyphenyl)-3-oxoprop-1-en-1-yl)-[1,1'biphenyl]-3-yl)-1-(4-hydroxy-3-methoxyphenyl)prop-2-en-1-one

A mixture of compound $40(0.26 \mathrm{~g}, 0.82 \mathrm{mmol})$, apocynin $(0.29 \mathrm{~g}, 1.8 \mathrm{mmol})$ and $\mathrm{LiOH}(0.41$ $\mathrm{g}, 9.8 \mathrm{mmol})$ in $\mathrm{MeOH}(10 \mathrm{ml})$ was subjected to microwave irradiation (power: $70 \mathrm{~W}$; temperature: $70^{\circ} \mathrm{C}$ ) for $30 \mathrm{~min}$ in a $30 \mathrm{~mL}$ glass pressure microwave tube. The reaction mixture was then acidified with $\mathrm{HCl} 10 \%$ solution and extracted with dichloromethane $(2 \times 30 \mathrm{~mL})$. The organic phase was dried over $\mathrm{Na}_{2} \mathrm{SO}_{4}$ and evaporated. Crude purification by flash chromatography using a 2:1 petroleum ether: acetone mixture as eluent gave product $\mathbf{2 9}$ as a 
yellow solid (0.2 g, 40\%): mp $204{ }^{\circ} \mathrm{C} ;{ }^{1} \mathrm{H}$ NMR $\delta 3.93(\mathrm{~s}, 6 \mathrm{H}), 4.78(\mathrm{~m}, 4 \mathrm{H})$, 5.33-5.37 (series of m, 2H), 5.51-5.56 (series of m, 2H), $6.22(\mathrm{~m}, 2 \mathrm{H}), 6.95(\mathrm{~d}, J=8.4 \mathrm{~Hz}, \mathrm{Ar}, 2 \mathrm{H}), 7.20(\mathrm{~d}, J=$ $8.8 \mathrm{~Hz}, \mathrm{Ar}, 2 \mathrm{H}), 7.70$ (d, $J=1.2 \mathrm{~Hz}, \mathrm{Ar}, 2 \mathrm{H}), 7.73$ (dd, $J=1.2 ; 10.8 \mathrm{~Hz}, \mathrm{Ar}, 2 \mathrm{H}), 7.81$ (dd, $J=$ $1.2 ; 10.0 \mathrm{~Hz}, \mathrm{Ar}, 2 \mathrm{H}), 8.05(\mathrm{~d}, J=16 \mathrm{~Hz}, 2 \mathrm{H}),(\mathrm{d}, J=8.8 \mathrm{~Hz}, \mathrm{Ar}, 2 \mathrm{H}), 8.2(\mathrm{~d}, J=16 \mathrm{~Hz}, 2 \mathrm{H})$, 8.22(d, $J=2.4 \mathrm{~Hz}, \mathrm{Ar}, 2 \mathrm{H}) ;{ }^{13} \mathrm{C}$ NMR $\delta 55.43,69.13,111.19,113.18,114.56,117.12,122.70$, 123.46, 124.51, 126.88, 129.64, 130.80, 132.91, 133.52, 137.84, 147.66, 151.42, 156.94, 187.37; Anal. Calcd. for $\mathrm{C}_{38} \mathrm{H}_{34} \mathrm{O}_{8}$ : C, 73.77; H, 5.54; Found C, 73.80, H, 5.57.

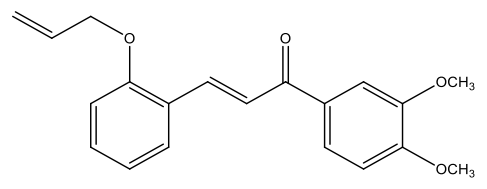

30

(E)-3-(2-(allyloxy)phenyl)-1-(3,4-dimethoxyphenyl)prop-2-en-1-one

To a solution of 2 (allyloxy) benzaldehyde [32] (1 g, $6.2 \mathrm{mmol}$ ) and 3,4-dimethoxy acetophenone $(1.1 \mathrm{~g}, 6.2 \mathrm{mmol})$ in $\mathrm{MeOH}(10 \mathrm{~mL})$ a solution of $\mathrm{LiOH}(0.89 \mathrm{~g}, 37.2 \mathrm{mmol})$ in $\mathrm{MeOH}(5 \mathrm{ml})$ was added dropwise. The reaction mixture was stirred at reflux for $12 \mathrm{~h}$. The solution was acidified with $\mathrm{HCl} 10 \%$ solution and extracted with dichloromethane $(2 \times 30 \mathrm{~mL})$. The organicphases were dried over $\mathrm{Na}_{2} \mathrm{SO}_{4}$ andconcentrated in vacuo. The crude product was purified by flash chromatography using dichloromethane as eluent to give product $\mathbf{3 0}$ as a white solid (1.25 g, 62\%): mp 87-88 ${ }^{\circ}$; ${ }^{1} \mathrm{H}$ NMR $\delta 3.95$ (s, $\left.3 \mathrm{H}\right), 3.96$ (s, $\left.3 \mathrm{H}\right), 4.61$ (d, J= 5.2 Hz, $2 \mathrm{H}), 5.26-5.47$ (series of $\mathrm{m}, 2 \mathrm{H}), 6.08(\mathrm{~m}, 1 \mathrm{H}), 6.88(\mathrm{~d}, J=8.0 \mathrm{~Hz}, \mathrm{Ar}, 1 \mathrm{H}), 6.97(\mathrm{t}, J=6.8 \mathrm{~Hz}$, Ar, 1H), 7.31 (dt, $J=1.6,4.8 \mathrm{~Hz}, \mathrm{Ar}, 1 \mathrm{H}$ ), 7.60-7.68 (series of m, Ar, 4H), 7.70 (d, $J=16 \mathrm{~Hz}$, $1 \mathrm{H}), 8.10(\mathrm{~d}, J=16 \mathrm{~Hz}, 1 \mathrm{H}) .{ }^{13} \mathrm{C}$ NMR $\delta 55.97,56.05,69.14,109.97,110.79,112.46,117.96$, 120.92 , 122.64, 122.97, 124.29, 129.58, 131.42, 131.54, 132.86, 139.65, 149.07, 153.02, 157.03, 189.14; Anal. Calcd. for $\mathrm{C}_{20} \mathrm{H}_{20} \mathrm{O}_{4}$ : C, 74.06; H, 6.22; Found: C, 74.16; H, 6.29.

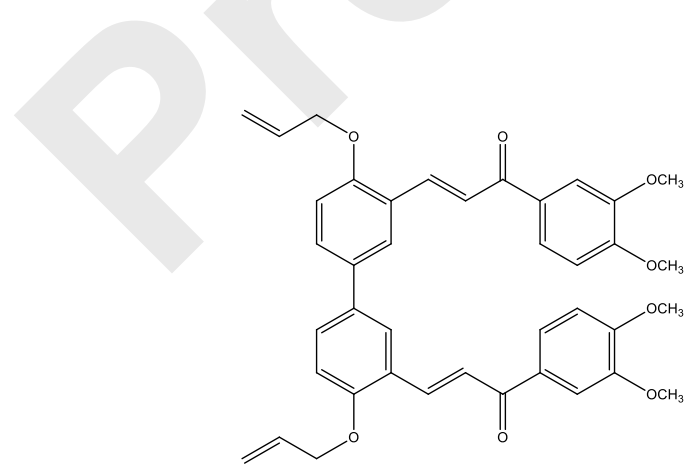

31

(2E,2'E)-3,3'-(4,4'-bis(allyloxy)-[1,1'-biphenyl]-3,3'-diyl)bis(1-(3,4-dimethoxyphenyl)prop-2en-1-one)

In a glass pressure microwave tube, a mixture of compound 40 (0.5 g, $1.6 \mathrm{mmol}), 3,4$ dimethoxyacetophenone $(0.6 \mathrm{~g}, 3.4 \mathrm{mmol})$ and $\mathrm{LiOH}(0.78 \mathrm{~g}, 18.6 \mathrm{mmol})$ in $\mathrm{MeOH}(10 \mathrm{ml})$ was subjected to microwave irradiation (power: $70 \mathrm{~W}$; temperature: $70^{\circ} \mathrm{C}$ ) and stirred for 30 min. The reaction mixture was then acidified with $\mathrm{HCl} 10 \%$ solution and extracted with dichloromethane $(2 \times 30 \mathrm{~mL})$. The organic solution was dried over $\mathrm{Na}_{2} \mathrm{SO}_{4}$ and evaporated. 
Purification by flash chromatography using a 7:3 petroleum ether: acetone mixture as eluent gave product 31 as a yellow solid. (0.16 g, 31\%): $\mathrm{mp} 145-146{ }^{\circ} \mathrm{C} ;{ }^{1} \mathrm{H}$ NMR $\delta 3.95$ (s, 3H), 3.97 $(\mathrm{s}, 3 \mathrm{H}), 4.70(\mathrm{~d}, J=5.6 \mathrm{~Hz}, 4 \mathrm{H}), 5.33-5.57$ (series of $\mathrm{m}, 4 \mathrm{H}), 6.22(\mathrm{~m}, 2 \mathrm{H}), 6.94(\mathrm{~d}, J=8.0 \mathrm{~Hz}$, Ar, 2H), 7.02 (d, $J=8.0 \mathrm{~Hz}, \mathrm{Ar}, 2 \mathrm{H}), 7.54(\mathrm{dd}, \mathrm{J}=2,8.4 \mathrm{~Hz}, \mathrm{Ar}, 2 \mathrm{H}), 7.64(\mathrm{~m}, \mathrm{Ar}, 2 \mathrm{H}), 7.71$ $(\mathrm{dd}, J=1.2,8.4 \mathrm{~Hz}, \mathrm{Ar}, 2 \mathrm{H}), 7.79(\mathrm{~d}, \mathrm{~J}=16 \mathrm{~Hz}, 2 \mathrm{H}), 7.82(\mathrm{~d}, \mathrm{~J}=2 \mathrm{~Hz}, \mathrm{Ar}, 2 \mathrm{H}), 8.14(\mathrm{~d}, J=$ $16 \mathrm{~Hz}, 2 \mathrm{H}), 8.22(\mathrm{~d}, J=2.4 \mathrm{~Hz}, \mathrm{Ar}, 2 \mathrm{H}) ;{ }^{13} \mathrm{C}$ NMR $\delta 56.04,56.07,69.42,110.01,110.85$, $112.95,118.16,123.09,123.20,124.71,127.97,129.65,131.51,132.80,133.16,139.58$, 149.17, 153.14, 157.13, 189.16; Anal. Calcd. for $\mathrm{C}_{40} \mathrm{H}_{38} \mathrm{O}_{8}$ : C, 74.29; H, 5.92; Found C, 74.35, H, 5.87 .<smiles>Cc1cc(-c2ccc(O)c(I)c2)ccc1O</smiles>

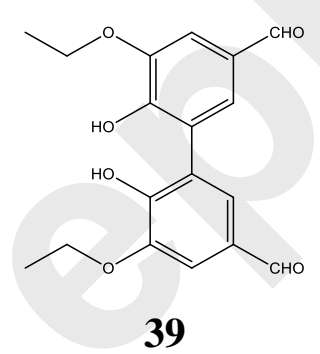

\section{5,5'-diethoxy-6,6'-dihydroxy-[1,1'-biphenyl]-3,3'-dicarbaldehyde}

In a glass pressure microwave tube, ethyl vanilline $(0.1 \mathrm{~g}, 0.6 \mathrm{mmol})$, iron sulfate heptahydrate $(0.0075 \mathrm{~g}, 0.03 \mathrm{mmol})$ and potassium peroxodisulfate $(0.081 \mathrm{~g}, 0.3 \mathrm{mmol})$ in water $(5 \mathrm{ml})$ were subjected, with stirring, to microwave irradiation (power: $100 \mathrm{~W}$; temperature: $110^{\circ} \mathrm{C}$ ) for 10 min. The reaction mixture was then acidified with $\mathrm{HCl} 10 \%$ solution and extracted with dichloromethane $(2 \times 30 \mathrm{~mL})$. The organic solution was dried over $\mathrm{Na}_{2} \mathrm{SO}_{4}$ and evaporated. The product was purified by chromatography on a column of silica gel with a mixture of $1: 4$ petroleum ether: ethyl acetate as eluent to give compound 39 as a yellow solid $(0.51 \mathrm{~g}, 52 \%)$ : mp 236-237 ${ }^{\circ} \mathrm{C} ;{ }^{1} \mathrm{H}$ NMR $\delta 1.49(\mathrm{t}, J=6.8 \mathrm{~Hz}, 6 \mathrm{H}), 4.23(\mathrm{q}, J=6.8 \mathrm{~Hz}, 4 \mathrm{H}), 6.6(\mathrm{bs}, 2 \mathrm{H}), 7.42$ $(\mathrm{d}, J=1.6 \mathrm{~Hz}, \mathrm{Ar}, 2 \mathrm{H}), 7.50$ (d, $J=1.6 \mathrm{~Hz}, \mathrm{Ar}, 2 \mathrm{H}) ;{ }^{13} \mathrm{C} \mathrm{NMR} \delta 14.71,65.13,108.71,113.89$, 120.76, 129.26, 131.48, 143.23, 190.48; Anal. Calcd.for $\mathrm{C}_{18} \mathrm{H}_{18} \mathrm{O}_{6}$ : C, 65.45; H, 5.49; Found: C, 65.50; H, 5.59 . 


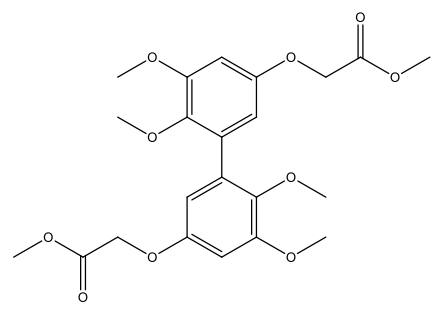

43

dimethyl 2,2'-((5,5',6,6'-tetramethoxy-[1,1'-biphenyl]-3,3'-diyl)bis(oxy))diacetate

To a solution of 5,5',6,6'-tetramethoxy-[1,1'-biphenyl]-3,3'-diol [14] (1.17 g, $3.8 \mathrm{mmol})$ and $\mathrm{K}_{2} \mathrm{CO}_{3}(2 \mathrm{~g}, 11.4 \mathrm{mmol})$ in dry DMF $(30 \mathrm{~mL})$, methyl bromoacetate $(1.50 \mathrm{~g}, 8.4 \mathrm{mmol})$ was added. The mixture was stirred at $\mathrm{rt}$ for $72 \mathrm{~h}$ and then water and $10 \% \mathrm{HCl}$ were added. The precipitate was filtered to obtain 43 as a yellow solid (1.62 g, 95\%): $\mathrm{mp} 132-133^{\circ} \mathrm{C} ;{ }^{1} \mathrm{H} \mathrm{NMR}$ $\delta 3.56(\mathrm{~s}, 6 \mathrm{H}), 3.78(\mathrm{~s}, 6 \mathrm{H}), 3.86(\mathrm{~s}, 6 \mathrm{H}), 4.57(\mathrm{~s}, 4 \mathrm{H}), 6.31(\mathrm{~d}, J=2.4 \mathrm{~Hz}, \mathrm{Ar}, 2 \mathrm{H}), 6.61(\mathrm{~d}, J=$ $2.4 \mathrm{~Hz}, \mathrm{Ar}, 2 \mathrm{H}) ;{ }^{13} \mathrm{C}$ NMR $\delta 52.21,55.84,60.74,65.60,100.92,106.34,132.27,141.53,153.51$, 153.54, 169.31; Anal. Calcd. for $\mathrm{C}_{22} \mathrm{H}_{26} \mathrm{O}_{10}$ : C, 58.66; H, 5.82; Found: C, 58.67; H, 5.79.

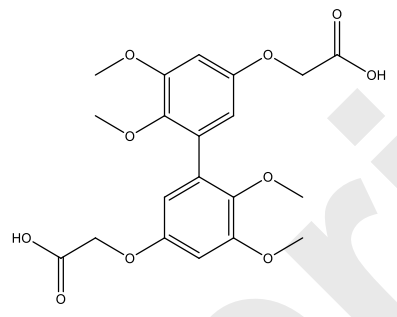

44

2,2'-((5,5',6,6'-tetramethoxy-[1,1'-biphenyl]-3,3'-diyl)bis(oxy))diacetic acid

To a solution of $43(1.32 \mathrm{~g}, 2.9 \mathrm{mmol}$ in dry THF (25 mL), lithium hydroxide $(0.50 \mathrm{~g}, 11.7$ mmol) was added under $\mathrm{N}_{2}$. The solution was stirred for $12 \mathrm{~h}$ at $70{ }^{\circ} \mathrm{C} .10 \% \mathrm{HCl}$ and water were added, the mixture was extracted with diethyl ether $(2 \times 50 \mathrm{~mL})$. The organic phases were dried and evaporated to get $\mathbf{4 4}$ as a white solid $(1.20 \mathrm{~g}, 98 \%)$ : $\mathrm{mp} 183-184^{\circ} \mathrm{C} ;{ }^{1} \mathrm{H}$ NMR $\delta 3.52$ (s, 6H), 3.83 (s, 6H), 4.67 (s, 4H), 6.37 (d, $J=2.1 \mathrm{~Hz}, \mathrm{Ar}, 2 \mathrm{H}), 6.63$ (d, $J=2.1 \mathrm{~Hz}, \mathrm{Ar}, 2 \mathrm{H})$; ${ }^{13} \mathrm{C}$ NMR $\delta$ 55.26, 59.73, 64.97, 100.32, 106.99, 132.99, 141.36, 153.55, 153.81, 169.46; Anal. Calcd.for $\mathrm{C}_{20} \mathrm{H}_{22} \mathrm{O}_{10}$ : C, 56.87; H, 5.25; Found: C, 56.79; H, 5.29.

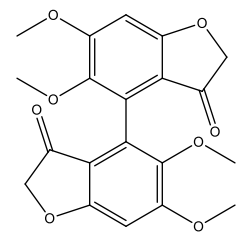

37

\section{5,5',6,6'-tetramethoxy-[4,4'-bibenzofuran $]-3,3^{\prime}\left(2 \mathrm{H}, 2^{\prime} \mathrm{H}\right)$-dione}

In a $25 \mathrm{~mL}$ flask, $8 \mathrm{~g}$ of polyphosphoric acid were weighed in and were heated at an oil bath temperature of $90^{\circ} \mathrm{C}$. Within $5 \mathrm{~min}$ compound $44(0.3 \mathrm{~g}, 0.7 \mathrm{mmol})$ was added; then stirring 
was carried out for $3 \mathrm{hrs}$ at $90^{\circ} \mathrm{C}$. The cooled solution was poured onto ice. After stirring during 2 hours, three extractions were carried out with a total of $400 \mathrm{~mL}$ of dichloromethane. The organic phases, washed with water and $10 \% \mathrm{~K}_{2} \mathrm{CO}_{3}$ solution, were dried over $\mathrm{Na}_{2} \mathrm{SO}_{4}$. After removal of the solvent, the residue was purified by chromatography on a column of silica gel using a 1:1 mixture of ethyl acetate:petroleum ether as eluent to get $\mathbf{3 7}$ as a yellow solid $(0.24$ g, 90\%): mp $138-140{ }^{\circ} \mathrm{C} ;{ }^{1} \mathrm{H}$ NMR $\delta 3.59$ (s, 6H), 3.92 (s, 6H), 4.52 (AB system, $J=17.6 \mathrm{~Hz}$, $4 \mathrm{H}), 6.65$ (s, Ar, 2H); ${ }^{13} \mathrm{C}$ NMR $\delta 56.24,60.96,75.45,96.07,111.35,124.01,142.56,161.24$, 172.43, 196.85; Anal. Calcd. for $\mathrm{C}_{20} \mathrm{H}_{18} \mathrm{O}_{8}$ : C, 62.18; H, 4.70; Found: C, 62.14; H, 4.56.

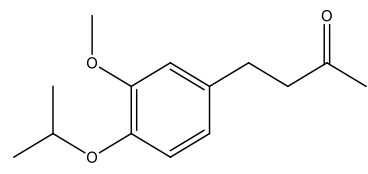

38

4-(4-isopropoxy-3-methoxyphenyl)butan-2-one

2-bromopropane (1.5 g, $12.4 \mathrm{mmol})$ was added to a mixture of compound $\mathbf{3}$ ( $2 \mathrm{~g}, 10.3 \mathrm{mmol}$ ) and $\mathrm{K}_{2} \mathrm{CO}_{3}(1.7 \mathrm{~g}, 12.4 \mathrm{mmol})$ in dry acetone $(30 \mathrm{~mL})$. The reation mixture was stirred at 60 ${ }^{\circ} \mathrm{C}$ for $12 \mathrm{hrs}$, filtered and evaporated. The resulting solid was treated with dichloromethane (50 $\mathrm{mL}$ ) and an $10 \% \mathrm{NaOH}$ acqueous solution $(30 \mathrm{~mL})$. The organic phase was dried over $\mathrm{Na}_{2} \mathrm{SO}_{4}$ and evaporated to get 38 as a colourless oil $(1.48 \mathrm{~g}, 61 \%) ;{ }^{1} \mathrm{H}$ NMR $\delta 1.31(\mathrm{~d}, J=6.4 \mathrm{~Hz}, 6 \mathrm{H})$, $2.10(\mathrm{~s}, 3 \mathrm{H}), 2.68-2.82($ series of $\mathrm{m}, 4 \mathrm{H}), 3.79(\mathrm{~s}, 3 \mathrm{H}), 4.44(\mathrm{sept}, J=6.4 \mathrm{~Hz}, 1 \mathrm{H}), 6.66(\mathrm{dd}, J=$ $2,8 \mathrm{~Hz}, \mathrm{Ar}, 1 \mathrm{H}), 6.68(\mathrm{~d}, J=2 \mathrm{~Hz}, \mathrm{Ar}, 1 \mathrm{H}), 6.77(\mathrm{~d}, J=8.0 \mathrm{~Hz}, \mathrm{Ar}, 1 \mathrm{H}) ;{ }^{13} \mathrm{C} \mathrm{NMR} \delta 22.10$, 29.36, 30.04, 45.35, 55.86, 71.51, 112.36, 116.24, 120.06, 134.13, 145.53, 146.16, 150.35, 208.11; Anal. Calcd. for $\mathrm{C}_{14} \mathrm{H}_{20} \mathrm{O}_{3}$ : C, 71.16; H, 8.53; Found: C, 71.20; H, 8.56.

Table I. Structural formulas of tested compounds and Oxy Score values

\section{Evaluation of the antioxidant potential (pro-oxidant/antioxidant activity) of the 36 compounds}

Healthy volunteers who had attended their regular medical check-up at the Military Medical Academy in Belgrade and had given approval that any serum left over after biochemical analyses planned by physicians could be used for this study. Fifty samples whose basic biochemical parameters were within metabolite reference ranges were selected. After thorough 
mixing the serum pool was aliquoted into $450 \mu \mathrm{L}$ portions and frozen at $-83^{\circ} \mathrm{C}$ until analyses took place.

The stock concentration of each of the compounds was $10 \mathrm{mg} / \mathrm{mL}$. DMSO was the solvent. All analyses were performed in triplicate. To $450 \mu \mathrm{L}$ of serum $50 \mu \mathrm{L}$ of each compound under investigation was added $(500 \mu \mathrm{L}$ total $)$ and then incubated at $37^{\circ} \mathrm{C}$ for $2 \mathrm{~h}$.

The same procedure was implemented at the samples with concomitant presence of tested substances and tert-butyl-hydroperoxide $(\mathrm{TBH})(0.5 \mu \mathrm{L} / \mathrm{mL}$ solution in distillate water) as prooxidant substance.

\section{Total Oxidative Potency (TOP)}

TOP was determined according to Erel [33] and Kotur-Stevuljevic et al. [34]. All oxidants in the sample (for example $\mathrm{H}_{2} \mathrm{O}_{2}$ and lipid hydroperoxides) oxidise a ferro-orthodianisidin complex to ferric ion in an acidic environment in the presence of glycerol. The resulting ferric ion forms a coloured complex with xylene-orange. Colour intensity is measured spectrophotometrically (A 560nm) and is proportional to the total content of oxidising molecules in the sample. The assay is calibrated with $\mathrm{H}_{2} \mathrm{O}_{2}(10-200 \mu \mathrm{mol} / \mathrm{L})$ and the results were expressed $\mu \mathrm{mol} \mathrm{H}_{2} \mathrm{O}_{2}$ Equiv/L.

\section{Pro-oxidant-Antioxidant Balance (PAB)}

The PAB indicates concomitant pro-oxidant load and antioxidative capacity of a particular organism. A modified version of a published method [35] was used for PAB determination. The assay determines the concentration of $\mathrm{H}_{2} \mathrm{O}_{2}$. The chromogen 3,3',5,5'-tetramethylbenzidine (TMB) reacts with both, $\mathrm{H}_{2} \mathrm{O}_{2}$ and antioxidants, (including uric acid and other reducing species). The reaction between $\mathrm{H}_{2} \mathrm{O}_{2}$ and chromogen is catalysed by the enzyme peroxidase, resulting in the oxidation of TMB to produce an intense colour. In contrast, the reaction between uric acid and similar compounds with chromogen is non-catalyzed not driven by peroxidase causing discolouration. The colour generated in the reaction is proportional to the ratio of prooxidants and antioxidants. Absorbance was read at $450 \mathrm{~nm}$ after a 10 minute incubation of the reaction medium at $37^{0} \mathrm{C}$.

\section{SH-groups (SHG)}

Total sulphydryl groups in serum was determined by a modification of Ellman's method [36] (according Kotur-Stevuljevic et al.) [34], based on the formation of a yellow-coloured reaction 
product between 2,2'-dinitro-5,5'-dithiobenzoic acid (DTNB) and aliphatic thiol compounds in basic conditions $(\mathrm{pH}=9.0)$. Absorbance was measured at $412 \mathrm{~nm}$.

\section{Total Antioxidant Capacity (TAC)}

TAC was measured using the stable $\mathrm{ABTS}^{+}$cation as a chromogen [37]. ABTS is oxidised by $\mathrm{H}_{2} \mathrm{O}_{2}$ in acetate buffer; $\mathrm{pH}=3.6$ to a green coloured $\mathrm{ABTS}^{+}$cation. Antioxidants present in the sample lead to varying degrees of discoloration proportional with their concentration (the antioxidant potential of the sample). After incubation for 10 minutes at room temperature absorbance at $600 \mathrm{~nm}$ was recorded.

\section{Pro-oxidative Score, Antioxidative Score and Oxy Score}

Overall oxidative stress was evaluated through several scores: Protective, damage and Oxy Score as previously suggested [38]. Antioxidative Score (indicating protective capacity) was calculated as the mean of the Z Scores of the measured antioxidant parameters - TAC and SHG. Pro-oxidative Score (indicating damaging potential) was calculated as mean Z Scores of the measured pro-oxidant parameters - TOP and PAB. Oxy Score was calculated as the difference between Pro-oxidative and Antioxidative Scores. Population parameters for $\mathrm{Z}$ scores calculation were from our previous investigations using a healthy population. The formula for $\mathrm{Z}$ score calculation was as follows: $(\mathrm{xi}-\overline{\mathrm{x}}) / \sigma$, where $\mathrm{xi}$ is sample value, $\overline{\mathrm{x}}$ - population mean, $\sigma$ - population standard deviation. A higher Oxy score indicates less antioxidative protection, in other words a more pronounced pro-oxidative state.

\section{Statistical analysis}

The results of all parameters were expressed as percentiles (medians with $25^{\text {th }}$ and $75^{\text {th }}$ percentile values). The non-parametric Kruskall-Wallis test with post hoc Mann-Whitney U test with Bonferroni correction was used for statistical analysis. Values of $\mathrm{p}<0.05$ were considered as statistically significant.

\section{Results}

The material for antioxidant potential assays comprises of 36 compounds (monomers or their C2-symmetric dimers) which synthesis started, for almostall of them, from naturally occurring compounds commercially available (Table I) and following methods known in literature or improving them by sustainable reagents and procedures. Compound $\mathbf{1}$ was prepared by condensation of 5,6-dimethoxybenzofuran-3(2H)-one with 4-methoxy-benzaldehyde in the 
presence of neutral alumina (with good yield). The same procedure was followed for the preparation of dimer $\mathbf{2}$, starting from the corresponding benzofuran-3-one dimer. Compound $\mathbf{5}$ was prepared in one pot (in $60 \%$ yield) by a coupling reaction of 4-(4-isopropoxy-3methoxyphenyl)butan-2-one in the presence of molybdenum (V) chloride. The coupling reaction of monomer $\mathbf{1 0}$ in the presence of methyl-tributylammonium permanganate at room temperature produced dimer $\mathbf{1 1}$ (in $61 \%$ yield). Claisen-Schmidt condensation of 5,5'-diethoxy6,6'-dihydroxy-[1,1'-biphenyl]-3,3'-dicarbaldehyde with acetone under basic conditions gave compound 13 (in $80 \%$ yield). Following the same reaction conditions, compound 21 was prepared starting from per-O-acetylated $\beta$-C-glucopyranosyl ketone and 5,5',6,6'-tetramethoxy[1,1'-biphenyl]-3,3'-dicarbaldehyde and further deacetylation (with $73 \%$ of overall yield). Following the same procedure, compound 20 was prepared (in $80 \%$ yield) starting from veratraldehyde. Compounds 14, 15, 17, 18, 19 and 40 were obtained by protection of the corresponding phenolic hydroxyl group with the appropriate organohalide under basic conditions, in acetone at reflux. The coupling reaction of raspberry ketone $\mathbf{2 2}$ in the presence of methyl-tributylammonium permanganate as catalyst gave dimer 23. Microwave procedures were applied for the preparation of compound dimers 25-29, 31 and monomer 26 following different reaction conditions. Dimer 25 was prepared (in 60\% yield) starting from the commercial 4,4'-dihydroxy biphenyl and hexamethylenetetramine in the presence of trifluoroacetic acid. A palladium catalysed Heck reaction between 3-methylpent-1-en-3-ol and 2-bromophenol under basic conditions gave compound 26. Following the same procedure for $\mathbf{2 6}$ and starting from the corresponding dimer dibromide, compound $\mathbf{2 7}$ was obtained (in $40 \%$ yield). Apocynin and 4,4'-bis(allyloxy)-[1,1'-biphenyl]-3,3'-dicarbaldehyde in the presence of lithium hydroxide in methanol produced dimer 29 (in $40 \%$ yield) after $30 \mathrm{~min}$ of microwave irradiation. Following the same procedure of 29, compound $\mathbf{3 1}$ was obtained (in $31 \%$ yield) starting from 4,4'-bis(allyloxy)-[1,1'-biphenyl]-3,3'-dicarbaldehyde and 3,4dimethoxyacetophenone. Monomer 30 was prepared (in $62 \%$ yield) following Claisen-Schmidt condensation of 3,4-dimethoxy-acetophenone and 2-(allyloxy)benzaldehyde under basic conditions.

Based on the values of the calculated Prooxidative scores and the calculated Antioxidative scores expressed together as Oxy score (Table I), six compounds were found to have strong antioxidant properties: 6, 7, 12, 13, 26, and 27 and four compounds were found to have extremely weak antioxidant properties: $\mathbf{2 , 2 9 , 3 0}$, and $\mathbf{3 1}$ in the serum pool assay. Table II shows 
results of all the distinct redox status markers measured in our current study using the in vitro assays.

\section{Table II Redox status parameters measured after the reaction of polyphenolic compounds with serum}

A group of weak antioxidants (compounds 2, 29-31) caused a significant increase in both PAB and TOP parameters compared to a group of strong antioxidants (compounds 6, 7, 12, 13, 26 and 27). Strong antioxidants significantly increased TAC values compared to weak antioxidants. After addition of exogenous pro-oxidant $\mathrm{TBH}$ in samples containing strong antioxidants, we noticed a decrease in TOP concentration for compounds $\mathbf{6 , 1 2}$ and $\mathbf{1 3}$, whereas for compounds 7 and 27 TOP concentration was unaffected. Compound 26 unexpectedly increased TOP. Because of obvious interactions between Ellman's reagents with polyphenolic compounds we omitted SHG from the calculation of the Antioxidative scores. When comparing the same parameters in the weak antioxidant compound group before and after TBH addition we found that PAB and TOP both diminished. All differences were significant $(\mathrm{p}<0.05)$. Unexpectedly TAC increased after TBH addition in the weak antioxidative compound group $(\mathrm{p}<0.05)$.

Regarding influence of TBH addition in samples of strong antioxidant groups we noticed significant increase in $\mathrm{PAB}$ values only for the $\mathbf{2 6}$ substance $(\mathrm{p}<0.05)$. PAB parameter remained at the basic level (without $\mathrm{TBH}$ ) for the other five strong antioxidants.

Trolox was used as a control, a known and characterized antioxidative substance. In our assay it's antioxidative activity was found to lie between that of the weak and strong groups of compounds. The Oxy Score values of the strong antioxidative compounds ranged from $\quad-28.8$ \pm 0.4 to $-32.5 \pm 1.1$, while the Oxy Score values of weaker antioxidative compounds were significantly more positive and ranged from $11 \pm 0.7$ to $14.6 \pm 0.8$. The Oxy Score for Trolox was -7.01 (-7.53- -6,51), so it was clearly positioned between strong and weak antioxidative compounds. There was a statistically significant difference in the Oxy Score between all strong antioxidative compounds and all weak antioxidative compounds $(\mathrm{p}<0.001)$. This was also true for strong antioxidative compounds andTroloxand weak antioxidative compounds and Trolox, both ( $\mathrm{p}<0.01$ ).Figures 1 and 2 show the results of 6 strongest antioxidative compounds and 4 weakest antioxidative compounds. The green line presents Trolox's value. Using the non- 
parametric Kruskall-Wallis and post hoc Mann-Whitney U test it was found that there was no statistically significant difference $(p>0.05)$ in the values of the Oxy Score (antioxidant activity capacity) of the 6 strongest antioxidative compounds [in assays without TBH (Fig. 1) and in those with TBH added (Fig. 2)].

Lipophilicity $(\log \mathrm{P})$ of the strongest antioxidative compounds in serum (compounds $\mathbf{6}, \mathbf{7}, \mathbf{1 2}$, 13 and 26) varied between 1.27 and 2.85 whereas higher LogPs value were calculated for the weakest antioxidative compounds (compounds $\mathbf{2}, \mathbf{2 9}, \mathbf{3 0}, \mathbf{3 1}$ ) in the range of $3.90-7.44$.

A similar trend was calculated in the series of antioxidants and pro-oxidants assayed without addition of human serum (Table I). Trolox's LogP value (3.19) was close to the series of compounds with the strongest antioxidative activity.

\section{Figure 1.}

\section{Figure 2.}

Results of in vitro analysis in samples in non-biological matrix (direct reaction of selected polyphenolic compounds with reagents for TAC, TOP and PAB determination, without addition of human serum), with a statistically significant difference between the parameters determined ( $p<0.001$ ), compounds $3,4,6,10,11$ and 12 were found to be the most effective antioxidants, while compounds with weakest antioxidant activity were 17, 19, 30 and 34 (Figure 3). There was no statistically significant difference in the group of the six newly selected potent antioxidants $(p>0.05)$. Figure 3 shows the obtained values of the six strongest antioxidative compounds and four weakest antioxidative compounds in analysis in samples in the absence of serum.

\section{Figure 3.}

Our results so far have identified the 6 strongest antioxidative compounds and the 4 weakest antioxidative compounds. Strongest: Dehydrozingeronederivates, monomers or dimers, [compound $\mathbf{6}$ and its structural analogue compound $\mathbf{1 2}$ (monomer), compounds $\mathbf{7}$ and $\mathbf{1 3}$ 
(dimers)], prenylated phenol and 4,4'-dihydroxybiphenyl derivative [compounds $\mathbf{2 6}$ (monomer) and 27 (dimer)]. Weakest: Aurone and chalconederivatives [compounds 2, 29 and $\mathbf{3 1}$ (dimers)] and one chalcone monomer (compound 30).

The strongest in vitro antioxidant activity in matrix in the absence of human serum was that of zingerone (compounds $\mathbf{3}$ and $\mathbf{4}$, monomer and dimer, respectively), dehydrozingerone (compound 6), an ethylvanyllin 3-buten-2-one derivative monomer (compound 12) and the vanillyl alcohol methyl esters monomer (compound 10) and dimer (compound 11). The weakest antioxidants was the dehydrozingeronedimer derivative (compound 17), a dimer of ethylvanylin 3-buten-2-one derivative (compound 19), a methylmagnolol derivative (compound 34) and a chalcone derivative (compound 30).

It is important to note that compounds $\mathbf{6}$ and $\mathbf{1 2}$ (strong antioxidative activity) and compound 30 (weak antioxidant activity) manifested equal activity both in the absence and presence of human serum.

\section{Discussion}

The pro-oxidant/antioxidant activity of polyphenolic compounds is dependent on various factors including metal-reducing potential, chelation properties, $\mathrm{pH}$, solubility and concentration [19]. Such factors together with polyphenolic compound bioavailability and stability in biological environments, need be considered when evaluating their potential antioxidant bioactivity $[17,40]$. There has been many polyphenolic compounds investigated so far for potential use in human medicine, but resveratrol became especially appreciated primarily for its antioxidant and anti-inflammatory potency. However, Chudzińska et al. concluded that its beneficial effects are still not clearly confirmed and that there is a need for the further and better controlled clinical studies [41]. On the other side, Patti et al. [42] confirmed beneficial effects of olive oil, as phenolic compound, on anthropometric and biochemical parameters, including inflammatory markers in patients with metabolic syndrome and hepatic steatosis. 
Molecules having two symmetric binding moieties bearing a flexible bridge of suitable length and properties, would be expected to show a higher target binding affinity leading to higher biological activity compared with molecules where one binding moiety is missing [21, 43, 44].

Due to their specific structure, hydroxylated biphenyls have been found to have better antioxidant effects than the corresponding monophenols [17, 39, 45]. The results obtained in our study confirm the finding that the strongest antioxidant effect in the (serum-containing) biological environment was achieved by compounds containing a free phenolic hydroxyl as an electron-donating group with a ethoxy/methoxy in orthoposition and the side chain with an $\alpha, \beta$ unsaturated ketone, which contributes to the stability of phenoxyl radicals by increasing electronic delocalisation in the bio-environment [18].

But interestingly, our results showed that dehydrozingerone monomer (compound 6) and ethylvanyllin 3-buten-2-one derivative (compound 12) exhibited better antioxidant effect than their corresponding dimers, even though an almost comparable Oxy Score was calculated for the corresponding dimers (compounds 7 and 13, respectively). The difference in the antioxidant activity of the dimers could have been due to the formation of intramolecular hydrogen bonds and reduced rotation (appearance of axial chirality) that hinders electronic delocalisation of the phenoxyl radical through the two non planar aromatic rings [46].

Vitamin E family, known as tocopherols, are methyl-substituted tocol derivatives. The most potent is $\alpha$-tocopherol (5,7,8-trimethyltocol) which contains three electron-donating methyl groups that increase the nucleophilicity and reactivity of the phenolic group at position 6 of the chromane ring. Additionally, resonance stabilization by the para oxygen in the chromane ring improves the stability of the $\alpha$-tocopherol radical [47].

One of the primary function of vitamin $\mathrm{E}$ is preventing the oxidation of lipids, particularly unsaturated fatty acids through its antioxidant effects. Vitamin $\mathrm{E}$ is a fat-soluble compound and penetrates biological membranes. Located in cell membranes, vitamin E could act toward reactive oxygen species (ROS), thus protecting cellular components from oxidative damage [47]. The $\alpha$-tocopherol donates its electrons to the free radicals to neutralize them, In this process, $\alpha$-tocopherol is fully oxidized to the $\alpha$-tocoquinone form and loses its antioxidant capacity.

Trolox is a water soluble analogue of $\alpha$-tocopherol, used as a reference in the evaluation of antioxidant activities of compounds [48]. In this work, Trolox was used as a positive control. 
Lipophilicity of Trolox and compounds 1-36 was estimated by ChemBioDraw Ultra 13.0 software (CambridgeSoft) expressed as $\log \mathrm{P}$ (the logarithm of the partition coefficient for $\mathrm{n}$ octanol/water) and listed in Table I. Although a carboxylic group is present in the structure of Trolox, the high $\log \mathrm{P}$ value of the compound (3.19) implies a lipophilic character, as already documented in another study [20]. The physicochemical property of Trolox would explain the high affinity of the compound towards cellular membranes [48], but also could elucidate Trolox affinity towards lipoprotein particles i.e. their lipid content.We could hypothesise a different partition of Trolox in the bilayer surface that influencesthe interactions of the compound with peroxyl radicals generated in a selected experimental method. This point offers an explanation for the medium antioxidativeTrolox activity positioned between strong and weak polyphenolic compounds analysed in this study.

An unexpectedly high antioxidant effect was exhibited by compound $\mathbf{2 6}$ which lacks in a guaiacyl unit and an $\alpha, \beta$-unsaturated methyl ketone side chain as in compounds $\mathbf{6}$ and 12. It is possible that both in serum, and under acidic or basic conditions, compound $\mathbf{2 6}$ could undergo elimination of water by proton removal from the methylene group of the allylic chain and subsequent formation of a prenylated unit that would stabilise the phenoxyl radical. Good antioxidant activity of this structural moiety was also confirmed in the corresponding dimer, compound 27.

Although there have been many studies in animal models using zingerone as antioxidant and anti-inflammatory compound [49], our results indicate that zingerone (compound $\mathbf{3}$ ) exhibited greater antioxidant activity in an assay without biological material (human serum).

Zingerone exhibits good radical-scavenging activity but poorly acts as a chain-breaking antioxidant in lipid auto-oxidation in comparison with dehydrozingerone $\mathbf{6}$ and its corresponding dimer 7. Lipophilic antioxidants play a crucial role in attenuating oxidative processes that occur in cell membranes in diseases including cancer and neurodegeneration [49$51]$.

We found that lipophilicity of the strongest antioxidants detected in human serum was lower in comparison to that of the weakest antioxidants which contained a protected alkyl phenolichydroxyl group. It is possible that hydrolytic enzymes present in human serum are not able to deprotect the alkylated phenolic-hydroxyl group that could occur in other biological system.

Raspberry ketone, compound $\mathbf{2 2}$, one of the main components of red berry fruits and responsible for inhibiting inflammatory processes $[43,54]$, exhibited a modest Oxy Score. The structure of 
compound 22, lacking in methoxyl group in ortho position to the phenolic-hydroxyl group and an $\alpha, \beta$-unsaturated lateral chain, did not achieve a calculated oxy score value as great as dehydrozingerone 6. Nevertheless, raspberry ketone has been considered a health-promoting compound and marked as food supplement [55].

Chalcones are a group of polyphenolic compounds that have recently been used as additives and ingredients in cosmetic preparations because of their potential high antioxidant and antiinflammatory effects [56,57]. However, the tested compounds in our study, which are monomers and dimers of chalcones and aurones (formed by cyclisation of chalcones), namely compounds 2, 29, 30 and 31, exhibited the weakest antioxidant properties. The reason for this is probably the small number and/or absence of free phenolic hydroxyl groups in the molecule (which are mandatory for the antioxidant action of polyphenols), their too high lipophilicity or their inadequate configuration.

Regarding part of results which showed comparison before and after TBH addition and opposite activity in a group of weak compared to strong antioxidants could be explained with the lack of any prooxidative capacity in weak antioxidants which could, according to Halliwell initiate triggering of fast and strong antioxidative response of biological medium [58,59].

\section{Conclusion}

From a diverse group of tested natural-like polyphenolic compounds comprising of zingerone, dehydrozingerone, aurone, chalcone, magnolol derivatives, monomers and their corresponding dimers, the greatest antioxidant activity was that of dehydrozingerone analogues (compounds 6 and 12). In the future we will focus on these two moieties to confirm their mechanism of antioxidant action. The results suggest that those compoundg could be candidates for the curcumin analogues that potentially improves its bioavailability in vivo. The most convincing confirmation of their antioxidative potency also comes from the results of the same activity achieved by the well-known antioxidant Trolox, which is a water-soluble vitamin E analogue. Trolox's activity was found between the strong and weak antioxidant compounds analysed in our study. This means that compounds $\mathbf{6}$ and $\mathbf{1 2}$ are worthy of further investigation in the antioxidant biology.

The inconsistency of our results regarding the antioxidant effects of the compounds in different matrices (human serum and in vitro environment without bio-matrix) supports the unpredictability of polyphenolic pharmacophore behaviour in a biological environment and the difficulty in elucidating the presumed mechanism of action. Selected dehydrozingerones would 
be good candidates for in-depth testing of their biological behaviour and for possible precursors for the synthesis of novel polyphenolic molecules for potential therapeutic applications. More studies (animal and human or cell culture based) are necessary to provide evidence and to elucidate dose-response and cost-benefit relationships between polyphenol-like compounds, their therapeutic potential and health benefits. One of the limitation of this current study is acelular material used (human serum), but this is available biomaterial upon which we have developed platform for testing different substances' interaction with human origin biomolecules.

\section{Conflicts of Interest}

The authors declare that there is no conflict of interest regarding the publication of this paper.

\section{Funding Statement}

This work was financially supported by grants from the Serbian Ministry of Education, Science and Technological Development (Project numbers 175035 and 172041) and was partially supported by grant from Sardinia Region, Italy (Project FSC 2014-2020, RASSR73282).

\section{Acknowledgments}

This work was financially supported by grants from the Serbian Ministry of Education, Science and Technological Development (Project numbers 175035 and 172041) and was partially supported by grant from Sardinia Region, Italy (Project FSC 2014-2020, RASSR73282). We thank to Dr. David R. Jones (Manchester, UK) for his excellent help in English language and critical revision of the manuscript.

The study funders had no role in study design, analysis, interpretation of data and writing the manuscript. The authors made the decision to submit the manuscript for publication.

\section{References}

1. Amorati R, Lucarini M, Mugnaini V, Pedulli GF. Antioxidant activity of $o$-bisphenols: the role of intramolecular hydrogen bonding. J Org Chem 2003; 68(13): 5198-204.

2. Foti MC. Antioxidant properties of phenols. J Pharm Pharmacol 2007; 59(12): 1673- 
85.

3. Nimse SB, Pal D. Free radicals, natural antioxidants, and their reaction mechanisms. RSC Adv 2015; 5: 27986-8006.

4. Giglio RV, Patti AM, Cicero AFG, et al. Polyphenols: Potential Use in the Prevention and Treatment of Cardiovascular Diseases. Curr Pharm Des 2018; 24(2): 239-58.

5. Cicero AFG, Colletti A, Bajraktari G, et al. Lipid lowering nutraceuticals in clinical practice: Position paper from an International Lipid Expert Panel. Arch Med Sci 2017; 13(5): 965-1005.

6. Cicero AFG, Ruscica M, Banach M. Resveratrol and cognitive decline: A clinician perspective. Arch Med Sci 2019; 15(4):936-43.

7. Kim DO, Ho JH, Young JK, Hyun SY, Lee CY. Sweet and sour cherry phenolics and their protective effects on neuronal cells. J Agric Food Chem 2005; 53(26): 9921-7.

8. Zahedipour F, Hosseini SA, Sathyapalan T, Majeed M, Jamialahmadi T, Al-Rasadi K, Banach M, Sahebkar A. Potential effects of curcumin in the treatment of COVID-19 infection. Phytother Res. 2020; 34(11): 2911-20.

9. Reddy RC, Vatsala PG, Keshamouni VG, Padmanaban G, Rangarajan PN. Curcumin for malaria therapy. Biochem Biophys Res Commun 2005; 326: 472-4.

10. Kumar L, Chhibber S, Harjai K. Zingerone inhibit biofilm formation and improve antibiofilm efficacy of ciprofloxacin against Pseudomonas aeruginosa PAO1. Fitoterapia. 2013; 73-8.

11. Profumo E, Buttari B, D'Arcangelo D, et al. The Nutraceutical Dehydrozingerone and Its Dimer Counteract Inflammation- and Oxidative Stress-Induced Dysfunction of In Vitro Cultured Human Endothelial Cells: A Novel Perspective for the Prevention and Therapy of Atherosclerosis. Oxid Med Cell Longev 2016; 2016: 1246485.

12. Mapoung S, Suzuki S, Fuji S, et al. Dehydrozingerone, a Curcumin Analog, as a 
Potential Anti-Prostate Cancer Inhibitor In Vitro and In Vivo. Molecules 2020; 25: 2737.

13. Quideau S, Deffieux D, Douat-Casassus C, Pouysegu L. Plant polyphenols: chemical properties, biological activities, and synthesis. Angew Chem Int Edit 2011; 50(3): 586621.

14. Ruzza P, Serra PA, Fabbri D, Dettori MA, Rocchitta G, Delogu G. Hydroxylated biphenyls as tyrosinase inhibitor: A spectrophotometric and electrochemical study. Eur J Med Chem 2017; 126: 1034-8.

15. Maioli M, Basoli V, Carta P, et al. Synthesis of magnolol and honokiol derivatives and their effect against hepatocarcinoma cells. PlosOne 2018; 13(2): e0192178.

16. Hajduk PJ, Bures M, Praestagaard J, Fesik SW. Privileged molecules for protein binding identified from NMR base screening. J Med Chem 2000; 43: 3443-7.

17. Fujisawa S, Atsumi T, Murakami Y, Kadoma Y. Dimerization, ROS formation, and biological activity of o-methoxyphenols. Arch Immunol Ther Exp 2005; 53: 28-38.

18. Baschieri A, Pulvirenti L, Muccilli V, Amorati R, Tringali C. Chain-breaking antioxidant activity of hydroxylated and methoxylatedmagnolol derivatives: the role of H-bonds. Org Biomol Chem 2017; 15: 6177-84.

19. Eghbaliferiz S, Iranshahi M. Prooxidant activity of polyphenols, flavonoids, anthocyanins and carotenoids: updated review of mechanisms and catalyzing metals. Phytother Res 2016; 30(9): 1379-91.

20. Socrier L, Rosselin M, Gomez Giraldo AM, et al. Nitrone-Trolox conjugate as an inhibitor of lipid oxidation: Towards synergistic antioxidant effects. Biomembranes 2019; 1861: 1489-1501.

21. Marchiani A, Mammi S, Siligardi G, et al. Small molecules interacting with $\alpha$ synuclein: antiaggregating and cytoprotective properties. Amino Acids 2013; 45: 327 - 
38.

22. Cook SP, Gaul C, Danishefsky SJ. En route to the total synthesis of tashironin: on the exercise of stereochemical control by a methyl group in mediating remote cyclization reactions. Tetrahedron Lett 2005; 46: 843-7.

23. Choi H, Ham SY, Cha E, et al. Structure-Activity Relationships of 6- and 8-Gingerol Analogs as Anti-Biofilm Agents. J Med Chem 2017; 60(23): 9821-37.

24. Tatsuzaki J, Bastow KF, Nakagawa-Goto K, Nakamura S, Itokawa H, Lee KH. Dehydrozingerone, Chalcone, and Isoeugenol Analogues as in vitro Anticancer Agents. J Nat Prod 2006; 69: 1445-9.

25. Varró G, Pálchuber P, Pogrányi B, et al. ( \pm )-trans-Dihydronarciclasine and $( \pm)$-transdihydrolycoricidine analogues modified in their ring A: Evaluation of their anticancer activity and a SAR study. Eur J Med Chem. 2019; 173: 76-89.

26. Dettori M.A, Fabbri D, Pisano M, Rozzo C. and Delogu G. Synthesis of hydroxylated biphenyls derivatives bearing an $\alpha, \beta$-unsaturated ketone as lead structure for the development of new drug candidates against malignant melanoma ChemMedChem 2020; 13: 1-16.

27. Oufensou S, Casalini S, Balmas V, et al. Prenylated trans-cinnamic esters and ethers against clinical Fusarium spp.: repositioning of natural compounds in antimicrobial discovery. Molecules 2021; 26: 658-75.

28. Lin CF, Hung CF, Aljuffali IA, Huang YL, Liao WC, Fang JY. Methylation and Esterification of Magnolol for Ameliorating Cutaneous Targeting and Therapeutic Index by Topical Application. Pharm Res 2016; 33: 2152-67.

29. Kong ZL, Tzeng SC, Liu YC. Cytotoxic neolignans: an SAR study. Bioorg Med Chem Lett 2005; 15: 163-6. 
30. Destwide C, Tinh NH, Mamlok L, Malthete J. Trioxatruxenes: A New Family of DiscLike Mesogens with a Complex Polymorphism. Mol Cryst Liq Cryst 1984; 114(1-3): 139-50.

31. Marques FA, Simonelli F, Oliveira ARM, Gohr GL, Leal PC. Oxidative coupling of 4substituted 2-methoxy phenols using methyltributylammonium permanganate in dichloromethane. Tetrahedron Lett 1998; 39: 943-6.

32. Khalil NSAM. Efficient synthesis of novel 1,2,4-triazole fused acyclic and 21-28 membered macrocyclic and/or lariat macrocyclic oxaazathia crown compounds with potential antimicrobial activity. Eur J Med Chem 2010; 45(11): 5265-77.

33. Erel O. A new automated colorimetric method for measuring total oxidant status. Clin Biochem 2005; 38: 1103-11.

34. Kotur-Stevuljevic J, Bogavac-Stanojevic N, Jelic-Ivanovic Z, et al. Oxidative stress and paraoxonase 1 status in acute ischemic stroke patients. Atherosclerosis 2015; 241: 192 8.

35. Alamdari DH, Paletas K, Pegiou T, Sarigianni M, Befani C, Koliakos G. A novel assay for the evaluation of prooxidant-antioxidant balance, before and after antioxidant vitamin administration in type II diabetes patients. Clin Biochem 2007; 40(3-4): 24854.

36. Ellman GL. Tissue sulphydryl groups. Arch Biochem Biophys. 1959; 82: 70-7.

37. Erel O. A novel automated direct measurement method for total antioxidant capacity using a new generation, more stable ABTS radical cation. Clin Biochem. 2004; 37: 277 85.

38. Veglia F. Werba JP. Tremoli E. et al. Assessment of oxidative stress in coronary artery bypass surgery: comparison between the global index OXY-SCORE and individual biomarkers. Biomarkers. 2009; 14(7): 465-72. 
39. Kancheva V, Slavova-Kazakova A, Fabbri D, et al. Protective effects of equimolar mixture of monomer and dimer of dehydrozingerone with alfa-tocopherol and/or ascorbyl palmitate during bulk lipid autoxidation. Food Chem 2014; 157: 263-74.

40. Decker EA. Phenolics: prooxidants or antioxidants? Nutr Rev 1997; 55: 396-407.

41. Chudzińska M, Rogowicz D, Wołowiec Ł, et al. Resveratrol and cardiovascular system-the unfulfilled hopes. Irish J Med Sci 2020; DOI: 10.1007/s11845-020-02441$\mathrm{x}$.

42. Patti AM, Carruba G, Cicero AFG, et al. Daily Use of Extra Virgin Olive Oil with High Oleocanthal Concentration Reduced Body Weight, Waist Circumference, Alanine Transaminase, Inflammatory Cytokines and Hepatic Steatosis in Subjects with the Metabolic Syndrome: A 2-Month Intervention Study. Metabolites 2020; 10(10): 392.

43. Pan J, Yin D, Ma L, Zhao Y, Zhao J, Guo L. Dimer and tetramer of gallic acid: facile synthesis, antioxidant and antiproliferative activities. Lett Drug Des Discov 2014; 11(1): 27-32.

44. Pani G, Dessì A, Dallocchio R, et al. Phenolic inhibitors of trichothecene biosynthesis by the wheat fungal pathogen Fusarium culmorum: a computational insight into the structure-activity relationship. PloS ONE 2016; 11: e0157316.

45. Slavova-Kazakova AK, Angelova SE, Veprintsev TL, et al. Antioxidant potential of curcumin-related compounds studied by chemiluminescence kinetics, chain-breaking efficiencies, scavenging activity (ORAC) and DFT calculation. Beilstein J Org Chem 2015; 11: 1398-1411.

46. Glunz PW. Recent encounters with atropisomerism in drug discovery. Bioorg Med Chem Lett 2018; 28(2): 53-60.

47. Wallace D, Lemke TL. Nutrition and Obesity. In: Roche VF, Zito SW, Lemke TL, 
Williams DA, editors. Foye's Principles of Medicinal Chemistry, $8^{\text {th }}$ edition. Wolters Kluwer, Philadelphia, USA; 2020. p. 1573-5.

48. Sagach VF, Scrosati M, Fielding J, Rossoni G, Galli C, Visioli F. The watersoluble vitamin $\mathrm{E}$ analogue Trolox protects against ischaemia/reperfusion damage in vitro and ex vivo. A comparison with vitamin E. Pharmacol Res 2002; 45: 435-9.

49. Kandemir FM, Yildirim S, Kucukler S, Caglayan C, Mahamadu A, Dortbudak MB. Therapeutic efficacy of zingerone against vancomycin-induced oxidative stress, inflammation, apoptosis and aquaporin 1 permeability in rat kidney. Biomed Pharmacother 2018; 105: 981-91.

50. Pappas A. Lipids and skin health. New Jersey, USA: Springer International Publishing; 2015.

51. Gurer-Orhan H, Ince E, Konyar D, Saso L, Suzen S. The role of oxidative stress modulators in breast cancer. Curr Med Chem 2018; 25: 4084-101.

52. Chang KH, Cheng ML, Chiang MC, Chen CM. Lipophilic antioxidants in neurodegenerative diseases. Clin Chim Acta 2018; 485: 79-87.

53. Jeong JB, Jeong HJ. Rheosmin, a naturally occurring phenolic compound inhibits LPS-inducers iNOS and COX-2 expression in RAW264.7 cells by blocking NF-kB activation pathway. Food Chem Toxicol 2010; 48: 2148-53.

54. Mohamed HE, Abo-Elmatty DM, Mesbah NM, Saleh SM, Ali AMA, Sakr AT. Raspberry ketone preserved cholinergic activity and antioxidant defense in obesity induced Alzheimer disease in rats. Biomed Pharmacother 2018; 107: 1166-74.

55. Kshatriya D, Li X, Giunta GM, et al. Phenolic-enriched raspberry fruit extract (Rubus idaeus) resulted in lower weight gain, increased ambulatory activity, and elevated hepatic lipoprotein lipase and heme oxygenase-1 expression in male mice fed a high-fat diet. Nutr Res 2019; 68:19-33. 
56. Gacche R, Khsirsagar M, Kamble S, et al. Antioxidant and anti-inflammatory related activities of selected synthetic chalcones: structure-activity relationship studies using computational tools. Chem Pharm Bull 2008; 56(7): 897-901.

57. Katsori AM, Hadjipavlou-Litina D. Recent progress in therapeutic application of chalcones. Expert Opin Ther Pat 2011; 21(10): 1575-96.

58. Halliwell B. Antioxidants paradox: less paradoxical now? Br J Clin Pharmacol 2013; 75(3): $637-44$.

59. Smith RE, Ozben T, Saso L. Modulation of oxidative stress: pharmaceutical and pharmacological aspects. Oxid Med Cell Longev 2019; 2019: 6380473. 
Table I Structural formulas of tested compounds and Oxy Score values

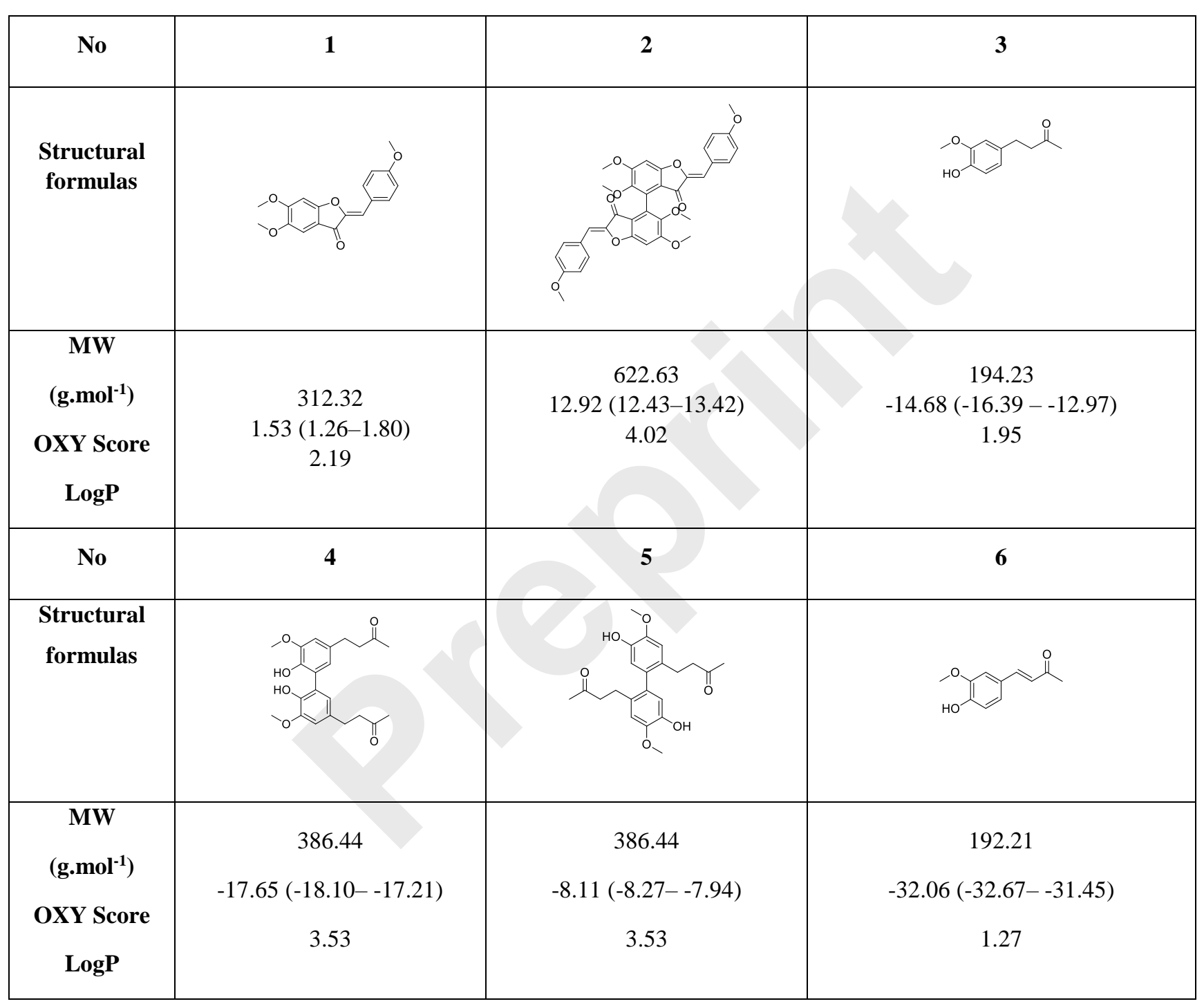




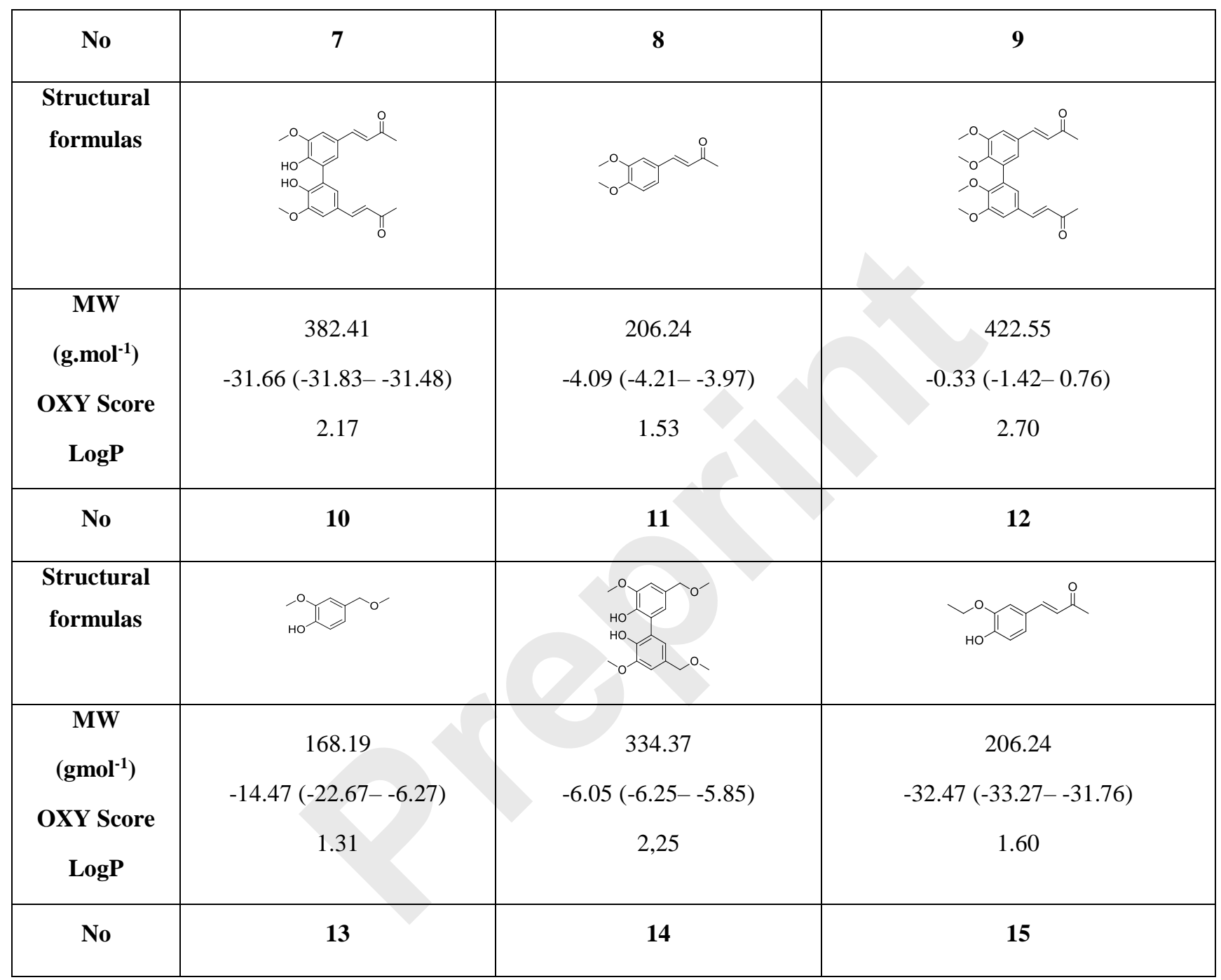




\begin{tabular}{|c|c|c|c|}
\hline $\begin{array}{l}\text { Structural } \\
\text { formulas }\end{array}$ & & & \\
\hline $\begin{array}{c}\text { MW } \\
\left(\text { gmol }^{-1}\right) \\
\text { OXY Score } \\
\text { LogP }\end{array}$ & $\begin{array}{c}410.47 \\
-32.04(-32.34--31.74) \\
2.85\end{array}$ & $\begin{array}{c}220.27 \\
-17.36(-18.33--16.40) \\
1.87\end{array}$ & $\begin{array}{c}438.52 \\
3.34(0.97--5.70) \\
3.37\end{array}$ \\
\hline No & 16 & 17 & 18 \\
\hline $\begin{array}{c}\text { Structural } \\
\text { formulas }\end{array}$ & & & \\
\hline $\begin{array}{c}\text { MW } \\
\left(\mathrm{gmol}^{-1}\right) \\
\text { OXY Score } \\
\text { LogP }\end{array}$ & $\begin{array}{c}220.27 \\
-3.94(-4.91--2.97) \\
1.87\end{array}$ & $\begin{array}{c}438.52 \\
-0.12(-0.78-0.54) \\
3.37\end{array}$ & $\begin{array}{c}234.30 \\
-6.45(-6.91--6.00) \\
2.20\end{array}$ \\
\hline No & 19 & 20 & 21 \\
\hline
\end{tabular}




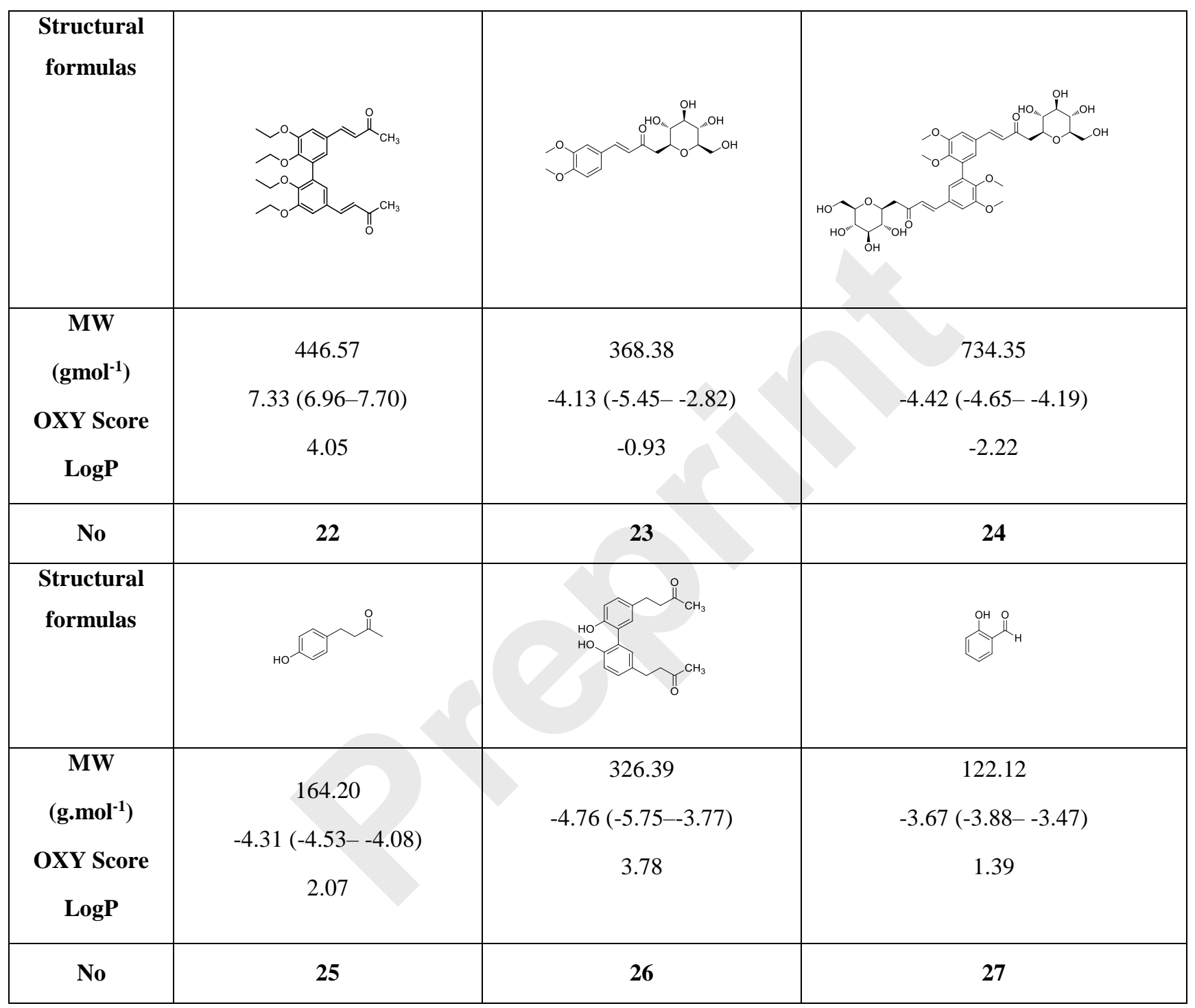




\begin{tabular}{|c|c|c|c|}
\hline $\begin{array}{l}\text { Structural } \\
\text { formulas }\end{array}$ & $\int_{\mathrm{OH}}^{\mathrm{O}}$ & & OH \\
\hline $\begin{array}{c}\text { MW } \\
\left(\text { g.mol }{ }^{-1}\right) \\
\text { OXY Score } \\
\text { LogP }\end{array}$ & $\begin{array}{c}242.23 \\
-0.92(-2.58-0.74) \\
2.42\end{array}$ & $\begin{array}{c}192.26 \\
-31.71(-32.03--31.40) \\
2.61\end{array}$ & $\begin{array}{c}382.50 \\
-28.85(-29.12--28.57) \\
4.86\end{array}$ \\
\hline No & 28 & 29 & 30 \\
\hline $\begin{array}{l}\text { Structural } \\
\text { formulas }\end{array}$ & & & \\
\hline $\begin{array}{c}\text { MW } \\
\left(\text { g.mol }^{-1}\right) \\
\text { OXY Score } \\
\text { LogP }\end{array}$ & $\begin{array}{c}310.35 \\
-2.48(-2.67--2.29) \\
3.63\end{array}$ & $\begin{array}{c}618.68 \\
11.55(10.64-12.47) \\
6.91\end{array}$ & $\begin{array}{c}324.38 \\
11.02(10.68-11.37) \\
3.90\end{array}$ \\
\hline No & 31 & 32 & 33 \\
\hline
\end{tabular}




\begin{tabular}{|c|c|c|c|}
\hline $\begin{array}{l}\text { Structural } \\
\text { formulas }\end{array}$ & & & \\
\hline $\begin{array}{c}\text { MW } \\
\left(\text { g.mol }^{-1}\right) \\
\text { OXY Score } \\
\text { LogP }\end{array}$ & $\begin{array}{c}646.74 \\
14.66(14.09-15.23) \\
7.44\end{array}$ & $\begin{array}{c}266.34 \\
-5.07(-5.72--4.42) \\
5.03\end{array}$ & $\begin{array}{c}280.37 \\
-1.73(-1.88--1.58) \\
5.30\end{array}$ \\
\hline No & 34 & 35 & 36 \\
\hline $\begin{array}{c}\text { Structural } \\
\text { formulas }\end{array}$ & & & \\
\hline $\begin{array}{c}\text { MW } \\
\left(\text { g.mol }^{-1}\right) \\
\text { OXY Score } \\
\text { LogP }\end{array}$ & $\begin{array}{c}294.39 \\
-1.23(-1.49--0.97) \\
5.56\end{array}$ & $\begin{array}{c}308.38 \\
-2.85(-3.56--2.14) \\
5.01\end{array}$ & $\begin{array}{c}350.41 \\
-10.67(-10.90--10.43) \\
4.99\end{array}$ \\
\hline
\end{tabular}




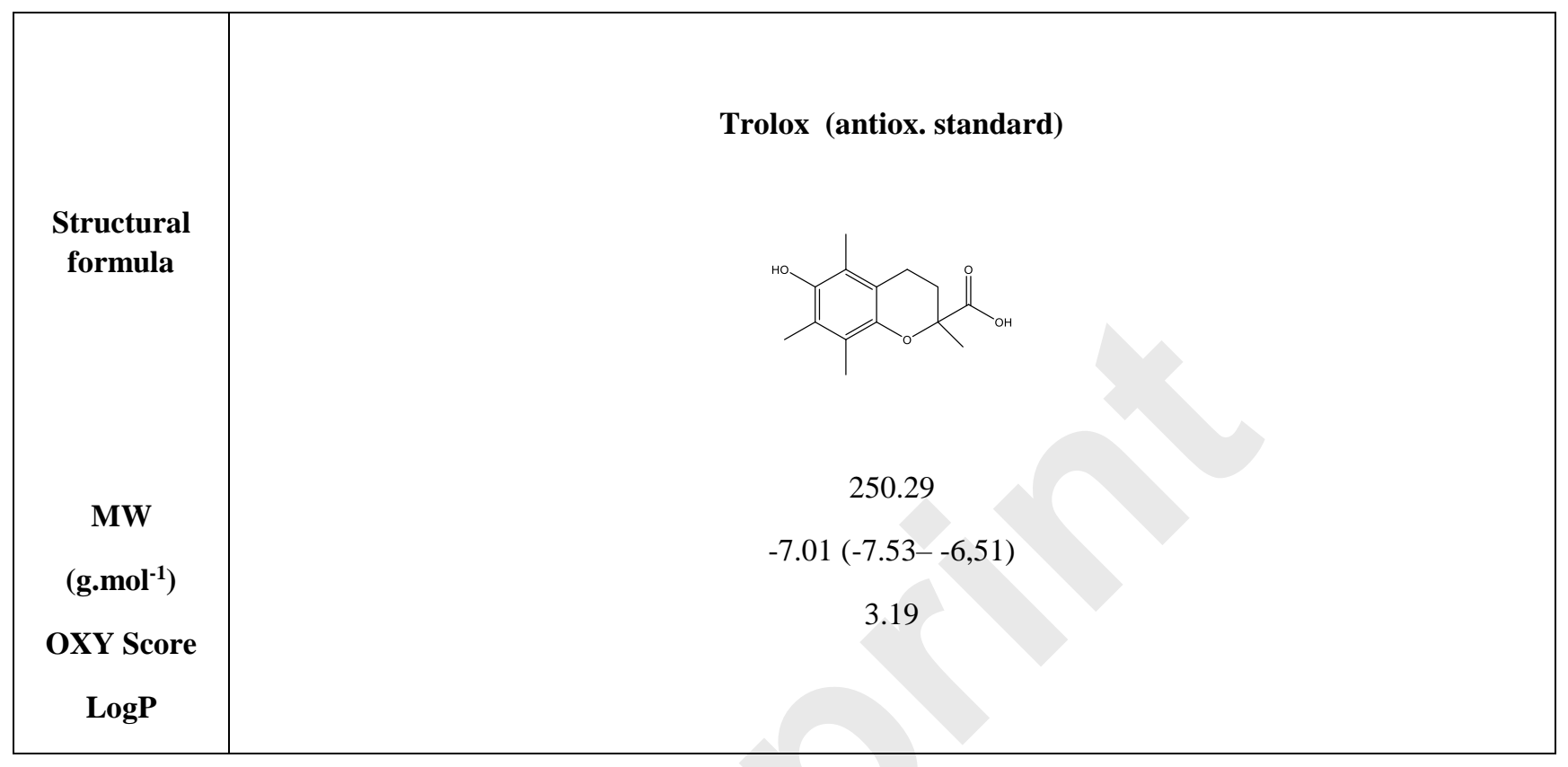

*Auron derivatives: compounds $\mathbf{1}$ (monomer) and $\mathbf{2}$ (dimer); zingerone derivatives: compounds 3 (zingerone, monomer), 4 (zingerone,dimer) and 5 (dimer); dehydrozingerone derivatives: compounds 6 (dehydrozingerone, monomer), 7 (dehydrozingerone,dimer), 8 (monomer) and 9 (dimer), 16 (OEt-dehydrozingerone, monomer), 17 (OEt-dehydrozingerone, dimer); vanillyl alcohol methyl esters: compounds 10 (monomer) and 11 (dimer); ethylvanylin 3-buten-2-one derivatives: compounds 12, 14 and 18 (monomers); 13,15 and 19 (dimers); glucosylateddehydrozingerone compounds: compounds 20 (monomer) and 21 (dimer); 4-(3-hydroxybutil-3-on) phenol (raspberry ketone): compounds 22 (raspberry ketone, monomer) and 23 (raspberry ketone, dimer); salicylaldehyde and its 4,4'-dihydroxybiphenyl derivative: compounds $\mathbf{2 4}$ (monomer) and 25 (dimer); prenylated phenol and 4,4'-dihydroxybiphenyl derivative: compounds 26 (monomer) and 27 (dimer); chalcones and 4,4'-dihydroxybiphenylchalcones: compounds $\mathbf{2 8}$ and $\mathbf{3 0}$ (monomers); 29 and $\mathbf{3 1}$ (dimers); magnolol: compound $\mathbf{3 2}$ (magnolol), and methylmagnolol derivatives: compounds $\mathbf{3 3}$ and $\mathbf{3 4}$; monoacetylmagnolol: compound 35, and diacetylmagnolol: compound $\mathbf{3 6}$.

**The solubility of all compounds was $15 \mathrm{mg} / \mathrm{mL}$ in DMSO (dimethyl sulfoxide), and concentrations of $10 \mathrm{mg} / \mathrm{mL}$ DMSO were used in this study for all compounds tested. 
Table II Redox status parameters measured after the reaction of polyphenolic compounds with serum

\begin{tabular}{|c|c|c|c|c|c|c|c|c|}
\hline $\begin{array}{l}\text { Polyphenolic } \\
\text { compound }\end{array}$ & $\begin{array}{l}\text { PAB } \\
(\mathrm{U} / \mathrm{L})\end{array}$ & TOP $(\mu \mathrm{mol} / \mathrm{L})$ & $\mathrm{SHG}(\mathrm{mmol} / \mathrm{L})$ & $\begin{array}{c}\text { TAC } \\
(\mu \mathrm{mol} / \mathrm{L})\end{array}$ & $\begin{array}{c}\text { PABtbh } \\
(\mathrm{U} / \mathrm{L})\end{array}$ & $\begin{array}{l}\text { TOPtbh } \\
(\mu \mathrm{mol} / \mathrm{L})\end{array}$ & SHGtbh (mmol/L) & TACtbh $(\mu \mathrm{mol} / \mathrm{L})$ \\
\hline \multirow[b]{2}{*}{2} & 127.5 & 87.5 & 3.2 & 2.2 & 113 & 47.6 & 2.00 & 152 \\
\hline & $(127.3-127.7)$ & $(82.3-92.7)$ & $(3.1-3.3)$ & $(1.0-4.2)$ & $(112-114)$ & $(45.7-49.6)$ & $(1.96-2.20)$ & $(42-263)$ \\
\hline \multirow[b]{2}{*}{6} & $15.9^{2}$ & $13.3^{2}$ & $1.0^{2}$ & $1588^{2}$ & $15.6^{2}$ & $6.45^{2}$ & $3.69^{2}$ & $1641^{2}$ \\
\hline & $(15.3-16.5)$ & $(9.8-16.9)$ & $(0.92-1.12)$ & $(1554-1621)$ & $(15.3-15.9)$ & $(4.42-20.4)$ & $(3.65-3.74)$ & $(1638-1644)$ \\
\hline \multirow[b]{2}{*}{7} & $15.3^{2}$ & $16.2^{2}$ & $3.7^{2,6}$ & $1415^{2}$ & $14.2^{2}$ & $16.87^{2}$ & $2.31^{6}$ & $1057^{2,6}$ \\
\hline & $(15.2-15.4)$ & $(15.3-17.2)$ & $(3.6-3.8)$ & $(1243-1587)$ & $(13.7-14.9)$ & $(13.5-20.20)$ & $(2.29-2.34)$ & $(867-1248)$ \\
\hline \multirow[b]{2}{*}{12} & $14.6^{2}$ & $13.2^{2}$ & $1.1^{2,7}$ & $1576^{2}$ & $13.8^{2}$ & $10.10^{2}$ & $3.68^{2,7}$ & $1696^{2}$ \\
\hline & $(13.6-15.5)$ & $(7.9-18.5)$ & $(1.0-1.2)$ & $(1562-1591)$ & $(12.2-15.5)$ & $(8.80-11.30)$ & $(3.38-4.00)$ & $(1642-1751)$ \\
\hline \multirow[b]{2}{*}{13} & $14.8^{2}$ & $17.7^{2,6,7,12}$ & $1.05^{2,7}$ & $1633^{2}$ & $12.5^{2}$ & $13.95^{2}$ & $3.34^{2,7}$ & $1554^{2}$ \\
\hline & $(14.7-15.0)$ & $(15.5-19.9)$ & $(1.0-1.1)$ & $(1614-1652)$ & $(12.4-12.7)$ & $(13.91-14.00)$ & (3.31-3.40) & $(1494-1615)$ \\
\hline \multirow[b]{2}{*}{26} & $16.0^{2}$ & $15.0^{2}$ & $0.98^{2,7}$ & $1502^{2}$ & $100^{2,6,7,12,13}$ & $22.06^{2,6}$ & $0.79^{2,6,7,12,13}$ & $1538^{2}$ \\
\hline & $(15.3-16.7)$ & $(13.7-16.3)$ & $(0.96-1.00)$ & $(1477-1527)$ & $(82-125)$ & $(21.68-22.42)$ & $(0.77-0.81)$ & $(1521-1555)$ \\
\hline \multirow[b]{2}{*}{27} & $20.5^{2}$ & $22.3^{2}$ & 1.29 & 1097 & $23.4^{2,26}$ & $20.81^{2,6}$ & $0.88^{2,6,7,12,13}$ & $966^{2,6,12,13,26}$ \\
\hline & $(20.3-20.8)$ & $(17.8-26.9)$ & $(1.16-1.42)$ & (956-1239) & $(21.8-25.0)$ & $(18.54-23.07)$ & $(0.84-0.92)$ & (953-978) \\
\hline \multirow[b]{2}{*}{29} & $125.8^{6,7,12,13,26,27}$ & $77.4^{6,7,12,13,26,27}$ & $3.4^{6,12,13}$ & $1.0^{6,7,12}$ & 114.8 & $42.84^{6,7,12,13,26,27}$ & $2.08^{6,26,27}$ & $130^{6,7,12,13,26,27}$ \\
\hline & $(124.3-127.3)$ & $(74.7-80.2)$ & $(3.3-3.6)$ & $(0.5-1.2)$ & $6,7,12,13,26,27$ & $(41.30-44.37)$ & $(2.06-2.10)$ & $(72-188)$ \\
\hline \multirow[b]{2}{*}{30} & 118.9 & $95.8^{6,7,12,13,26,27}$ & $2.1^{2,6,7,12,13}$ & $7.1^{6,7,12}$ & $102.7^{2,6,7,27,29}$ & $31.41^{6,7,12,13,26,27}$ & $0.99^{2,6,7,29}$ & $311^{6,7,12,13,26,27}$ \\
\hline & $2,6,7,12,13,26,27,29$ & $(95.3-96.3)$ & $(2.0-2.2)$ & $(0.4-13.8)$ & (101.9-103.5) & $(20.02-42.80)$ & $(0.95-1.1)$ & $(298-324)$ \\
\hline \multirow[b]{2}{*}{31} & 130.7 & $95.4^{6,7,12,13,26,27}$ & $2.41^{2,6,7,12,13}$ & $16.7^{6,7,12}$ & 117.2 & $60.80^{6,712,13,26}$ & $0.82^{6,7,26}$ & $314^{6,7,12,13,26,27}$ \\
\hline & $6,7,12,13,26,27,29,30$ & (91.1-99.7) & $(2.39-2.44)$ & $(2.5-65.8)$ & $6,7,12,13,26,27,30$ & $(47.42-74.09)$ & $(0.78-0.93)$ & $(288-340)$ \\
\hline
\end{tabular}


112.8

0.51

84.57

$2,6,7,12,13,26,27,29,30,31$

$2,6,7,12,13,26,27,29,30,31$

(109.7-115.7)

0.049

0.035

(0.50-0.52)

0.077

0.035
96.03

$6,7,12,13,27$

125.65

$2,6,7,12,13,26,27,29,30,31$

752

$2,6,7,12,13,26,27,29,30,31 \quad 2,6,7,12,13,26,27,29,30,31$

$p$

0.041

(95.43-96.63)

(123.73-127.58)

(0.44-0.52)

(733-771)

0.038

p-Kruskal-Wallis test; superscript numbers above median values means significant difference from distinct polyphenolic compound 


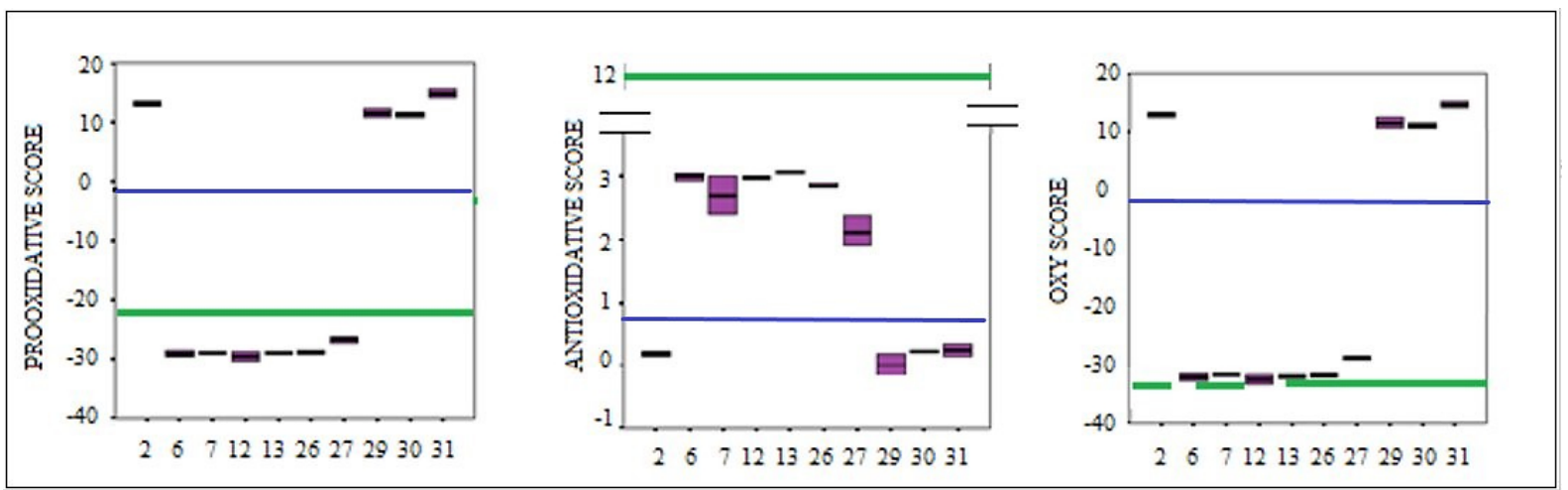

Figure 1. The values of Pro-oxidative, Antioxidative, and Oxy Scores of six strong (compounds 6, 7, 12, 13, 26, 27) and four weak (compounds 2, 29, 30, 31) antioxidants in the serum matrix without TBH addition. The green solid line represents the value obtained with Trolox (standard), the blue solid line represents values obtained with serum with DMSO as solvent (control). 

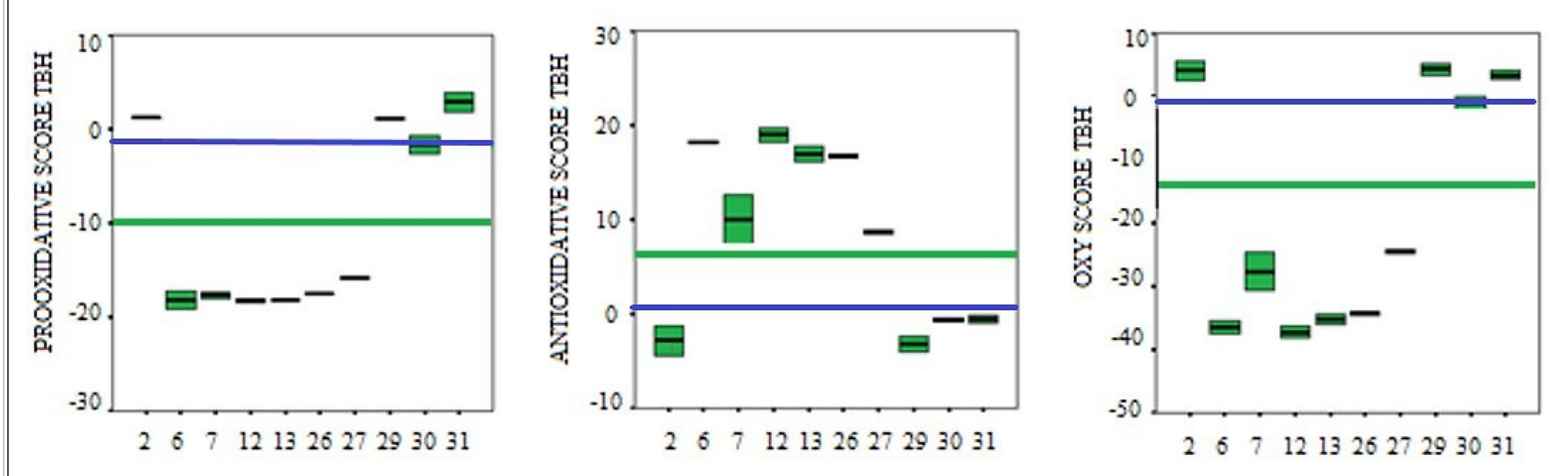

Figure 2. The values of Pro-oxidative, Antioxidative, and Oxy Scores of six strong (compounds 6, 7, 12, 13, 26, 27) and four weak (compounds 2, 29, 30, 31) anti-oxidants in the serum matrix with TBHaddition. The green solid line represents the value obtained with Trolox (standard), the blue solid line represents values obtained with serum with DMSO as solvent (control). 


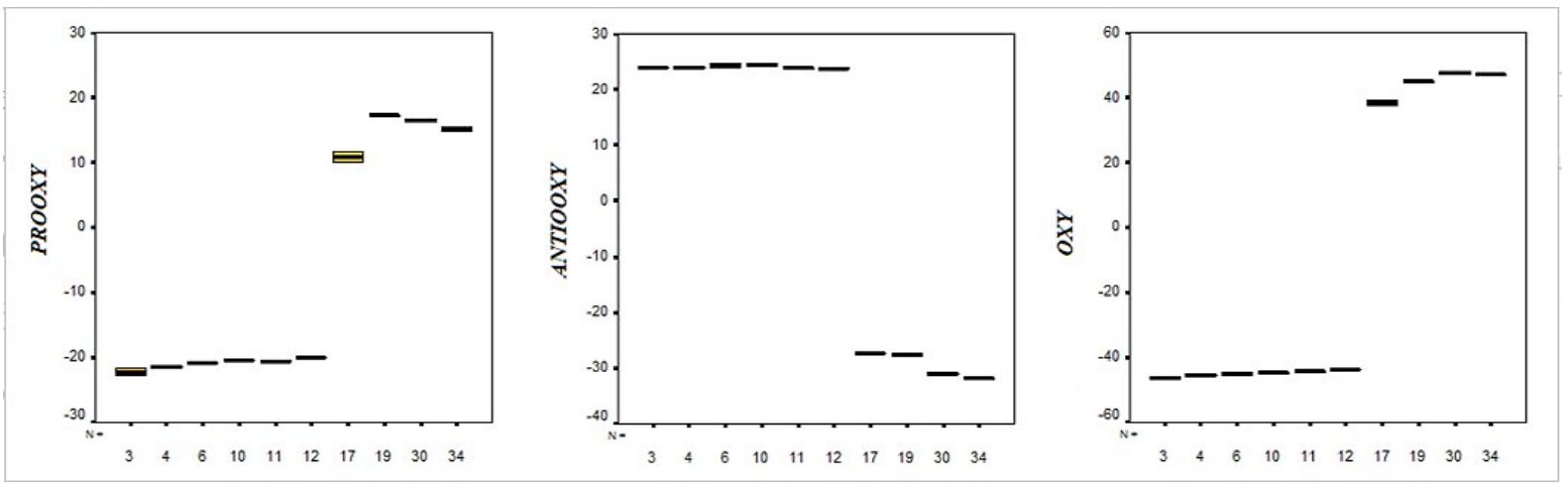

Figure 3. The values of Pro-oxidative, Antioxidative, and Oxy Scores of the 6 strongest antioxidative compounds $(3,4,6,11,12)$ and the 4 weakest antioxidative compounds $(17$, $19,30,34)$ in samples without addition of human serum. 